\title{
The spiral wind-up and dissipation of vorticity and a passive scalar in a strained planar vortex
}

\author{
By ANDREW P. BASSOM AND ANDREW D. GILBERT \\ School of Mathematical Sciences, University of Exeter, North Park Road, \\ Exeter, Devon EX4 4QE, UK
}

(Received 21 July 1998 and in revised form 6 April 1999)

The response of a Gaussian vortex to a weak time-dependent external strain field is studied numerically. The cases of an impulsive strain, an on-off step function, and a continuous random strain are considered. Transfers of enstrophy between mean and azimuthal components are observed, and the results are compared with an analogous passive scalar model and with Kida's elliptical vortex model.

A 'rebound' phenomenon is seen: after enstrophy is transferred from mean to azimuthal component by the external straining field, there is a subsequent transfer of enstrophy back from the azimuthal component to the mean. Analytical support is given for this phenomenon using Lundgren's asymptotic formulation of the spiral wind-up of vorticity. Finally the decay of the vortex under a continuous random external strain is studied numerically and compared with the passive scalar model. The vorticity distribution decays more slowly than the scalar because of the rebound phenomenon.

\section{Introduction}

Simulations of two-dimensional turbulence, beginning with random small-scale initial conditions, show that the vorticity distribution tends to organize into a number of coherent vortices, which then dominate the fluid flow (see for example Fornberg 1977; McWilliams 1984; Brachet et al. 1988). These vortices have a high Reynolds number $R e$ and tend to be isolated, there being a disparity between the length scale $l$ of a typical vortex and that of the separation $L$ between vortices. On the large scale $L$ the vortices move under their mutual interactions, and at leading order are governed by the dynamics of a number of point vortices (excepting collisions) (Ting \& Klein 1991; Lingevitch \& Bernoff 1995). On the moderate scale $l$, an individual vortex can be considered an approximately axisymmetric distribution of vorticity immersed in the time-dependent irrotational flow generated by the remaining vortices (cf. Oetzel \& Vallis 1997). As far as the internal dynamics of the vortex are concerned, the leading effect of neighbouring vortices is to impose a time-dependent straining field of magnitude $\varepsilon=l^{2} / L^{2} \tau$, varying over a time scale $1 / \varepsilon$, where $\tau$ is the turnover time of an individual vortex.

In this paper we study numerically the response of a vortex to different types of external straining field. We quantify the transfers of enstrophy between mean and fluctuating components, and so the losses of enstrophy to dissipation. Our aim is to understand the robustness of such vortices, as seen in two-dimensional turbulence and in related geophysical situations (see for example Smith \& Montgomery 1995). 
The inviscid equilibrium states of an elliptical vortex patch (with vorticity constant inside and zero outside) in an external strain field, and their stability, have been studied by Moore \& Saffman (1971). Equilibrium states with viscosity in two and three dimensions have been discussed by Robinson \& Saffman (1984), Moffatt, Kida \& Ohkitani (1994), Jiménez, Moffatt \& Vasco (1996) and Kevlahan \& Farge (1997); in these cases the leading-order structure of the vortex is viscously controlled and the time scale for such a vortex to equilibrate is correspondingly long. The emphasis in the present paper is rather different, being on transient, time-dependent phenomena, and on the behaviour at high Reynolds number, or even in an inviscid vortex. The closest dynamical model to ours is that of Kida (1981). Here an elliptical vortex patch evolves inviscidly under its own flow field and a time-dependent external strain field. The vortex always remains elliptical in form, the lengths and orientation of the principal axes of the ellipse being governed by ordinary differential equations. Solutions to these equations include the equilibria of Moore \& Saffman (1971), rotating, nutating ellipses, and indefinite extension of an ellipse in the strain field (Kida 1981; Dritschel 1990).

While Kida's model is very attractive, the reversible, 'elastic' behaviour of an elliptical vortex patch is rather special. Such vortices cannot show the process of relaxation to axisymmetry which commonly $\dagger$ occurs for smooth vortices (for example McCalpin 1987; Melander, McWilliams \& Zabusky 1987; Smith \& Montgomery 1995; Yao \& Zabusky 1996). As an instance of such 'axisymmetrization', consider a Gaussian vortex exposed to an impulsive external strain field (Bernoff \& Lingevitch 1994, henceforth referred to as BL94). An azimuthal $n=2$ mode is generated, which rapidly evolves into spiral arms tightly wound around the vortex. Once the azimuthal vorticity is of sufficiently small scale, the corresponding stream function is subdominant, and the vorticity behaves approximately as a passive scalar.

If weak viscosity is present the azimuthal component is eventually destroyed on a 'shear-diffuse' time scale of order $R e^{1 / 3} \tau$ (Lundgren 1982; Rhines \& Young 1982; BL94). The corresponding time scale for a passive scalar in closed stream lines is $O\left(P e^{1 / 3} \tau\right)$, where $P e$ is the Péclet number (Weiss 1966; Moffatt \& Kamkar 1983; Rhines \& Young 1983). The generation of azimuthal structure and its destruction on the shear-diffuse time scale represents an irreversible process that destroys enstrophy. This is not present in the inviscid Kida vortex model; while a smooth viscous vortex should exhibit some of the features of the Kida model, we would expect the elastic behaviour to become damped.

Although consideration of the action of viscosity highlights this loss of enstrophy through the shear-diffuse mechanism, the essential irreversible process is present even if the fluid is inviscid. In such a fluid a Gaussian vortex in an impulsive strain field again generates spiral arms of vorticity that are then driven to fine scales. With $R e=\infty$, such vorticity fluctuations are never destroyed pointwise; however in an average sense the fluctuations and corresponding enstrophy are lost from the largescale flow. This was quantified in Bassom \& Gilbert (1998, henceforth referred to as BG98), by considering the behaviour of spatial averages of the azimuthal vorticity field. Such 'weak' measures of the fluctuation field decay in time because of its progressive reduction in scale. In BG98 the decay rates were obtained, and differences

$\dagger$ Note that vortices do not always relax to axisymmetry; persistent nonlinear, non-axisymmetric states are seen by Dritschel (1989a, 1998), Koumoutsakos (1997) and Rossi, Lingevitch \& Bernoff (1997). As far as we are aware, the issue of whether linear perturbations with $n \geqslant 2$ relax to axisymmetry in the unbounded plane is unresolved (see Briggs, Daugherty \& Levy 1970; Smith \& Rosenbluth 1990; Bernoff \& Lingevitch 1994; Llewellyn Smith 1995). 
were found between the wind-up of vorticity and of a passive scalar, because of a residual coupling of vorticity to the stream function near the origin.

From this point of view, weak viscosity acts merely as a fine-scale cut-off in destroying azimuthal components through the fast shear-diffuse mechanism. The real irreversible process is the essentially inviscid generation of fine-scale structure in the first place: in the presence of the large-scale flow of the vortex, such structure is lost from the large scales, whether or not it is finally dissipated by viscosity, hyperdiffusion or contour surgery, or cascades indefinitely for $R e=\infty$. The amount of enstrophy that is lost can also be calculated without consideration of viscosity. Similar ideas underly the statistical approach of Robert \& Sommeria (1991).

The study BG98 of the response of a Gaussian vortex to an impulsive strain was entirely linear; in the present paper we consider the nonlinear response to several types of external strain: an impulse, a step function, and a random function of time. In $\S 2$ we set up the equations governing vorticity in a weak external flow field, with magnitude governed by the small parameter $\varepsilon$. These describe the generation of azimuthal vorticity at order $\varepsilon$, and the interaction between mean and azimuthal fields at order $\varepsilon^{2}$. We also set up an analogous problem in which a passive scalar evolves in a prescribed flow. Such problems form an important subject in their own right (e.g. Aref 1984; Ottino 1989) and we shall say little that is new from this point of view. However the passive scalar model provides a useful point of comparison for the numerical results for vorticity (cf. Babiano et al. 1987; Ohkitani 1991).

We present numerical simulations of the response to an impulsive straining flow in $\S 3$, typical of a short correlation-time input. By comparison with a scalar field, relatively small amounts of enstrophy are lost in the process of spiral wind-up, there being a transfer of enstrophy back from azimuthal to mean components. This transfer is also implicit in the results of BG98, and is particularly noticeable near the centre of the vortex. A long correlation-time strain is considered in $\S 4$ : a constant strain is switched on, and over many turnover times the vortex relaxes to a strained state described by the asymptotics of Moffatt et al. (1994) and Jiménez et al. (1996). The strain field is then switched off and the vortex relaxes once more to axisymmetry.

These studies of very simple external inputs complement $\S 5$, in which we introduce a weak time-dependent random strain; this is closest to what a vortex would experience in two-dimensional turbulence. We compare how vorticity and scalar distributions spread and dissipate. Finally $\S 6$ offers conclusions and discussion. Our philosophy in this paper, as in BG98, is as far as possible to work with $R e=\infty$. Since our study is primarily numerical it is necessary to introduce some level of diffusion in all our simulations; however our aim is to establish the important features of inviscid behaviour. We give further comments on the role of viscous diffusion as the paper proceeds.

\section{Governing equations and invariants}

\subsection{Vorticity problem}

A vortex with vorticity $\omega$ and stream function $\psi$ in an externally imposed irrotational flow with stream function $\psi_{\text {ext }}$ is governed by the dimensionless equations

$$
\begin{gathered}
\partial_{t} \omega=J\left(\psi+\psi_{\text {ext }}, \omega\right)+R e^{-1} \nabla^{2} \omega, \\
\nabla^{2} \psi=-\omega, \quad \nabla^{2} \psi_{\text {ext }}=0,
\end{gathered}
$$

where $J(a, b) \equiv r^{-1}\left(\partial_{r} a \partial_{\theta} b-\partial_{\theta} a \partial_{r} b\right)$. The two stream functions, $\psi$ and $\psi_{\text {ext }}$, are distinguished by their behaviour at infinity. The stream function $\psi$ of the vortex is 
determined by inverting (2.2a) using the integral

$$
\psi(\boldsymbol{r})=-\frac{1}{2 \pi} \int \omega(\boldsymbol{s}) \log |\boldsymbol{r}-\boldsymbol{s}| \mathrm{d}^{2} \boldsymbol{s}
$$

and grows as $\psi \sim-(\gamma / 2 \pi) \log r$ as $r \rightarrow \infty$, where $\gamma$ is the total circulation of the vortex. It is natural to take the non-dimensionalization in (2.1) above so as to make the circulation $\gamma$ and the length scale $l$ of the vortex unity.

The external stream function $\psi_{\text {ext }}$ is assumed to be generated outside the vicinity of the chosen vortex, by distant vortices or moving boundaries, and is a harmonic function, having algebraic growth away from the vortex. Near the vortex $\psi_{\text {ext }}$ can be expanded as a sum of decreasing terms $r^{n} \mathrm{e}^{\mathrm{e} \mathrm{i} n \theta}$, for $n \geqslant 0$. The constant $(n=0)$ term has no effect while the next, $n=1$, term gives a uniform flow, not of interest to us here (but see Lingevitch \& Bernoff 1995). The next term, $n=2$, is an external straining of the vortex and is the dominant effect on the internal dynamics of the vortex; we therefore restrict investigation to an external flow with only $n=2$ modes and write

$$
\psi_{\mathrm{ext}}=\varepsilon q(t) \phi(r) \mathrm{e}^{\mathrm{i} n \theta}+\text { c.c., } \quad \phi(r) \equiv r^{n} \quad(n=2) .
$$

Here 'c.c.' denotes the complex conjugate of the preceding expression and to bring out the general structure of equations we present, we leave $n$ in explicitly even though it is understood that our focus is on $n=2$.

The quantity $q(t)$ above is a complex function of time that determines the external strain; the modulus $|q|$ gives the strength of the strain, while the axes of the strain are oriented at an angle $\pi / 4-(\arg q) / 2$ to the usual Cartesian axes. This allows for fixed axes (as in $\S \S 3,4)$, randomly varying axes $(\S 5)$, or steadily rotating axes (see, for example, Kida 1981 or Dritschel 1990). (Note however that in contrast with these references, we always work in a frame with irrotational fluid about the vortex, never a rotating frame.)

Since the external flow is weak, in $(2.4 a, b) \varepsilon$ is a small parameter with $0<\varepsilon \ll 1$. In $\S \S 2-4$ we expand vorticity in powers of $\varepsilon$ as follows:

$$
\omega=\omega_{0}(r, t)+\varepsilon \omega_{1}(r, t) \mathrm{e}^{\mathrm{in} \theta}+\text { c.c. }+\varepsilon^{2}\left(\omega_{2}(r, t)+\omega_{22}(r, t) \mathrm{e}^{2 i n \theta}+\text { c.c. }\right)+\cdots
$$

and similarly for $\psi$. When these are substituted into the governing equations (2.1), (2.2) we obtain

$$
\begin{array}{ll}
\partial_{t} \omega_{0}=R e^{-1} \Delta_{0} \omega_{0}, & -\omega_{0}=\Delta_{0} \psi_{0}, \\
\partial_{t} \omega_{1}+\mathrm{i} n \alpha \omega_{1}+\mathrm{i} n \beta\left(\psi_{1}+q \phi\right)=R e^{-1} \Delta_{1} \omega_{1}, & -\omega_{1}=\Delta_{1} \psi_{1}, \\
\partial_{t} \omega_{2}+\mathrm{i} n r^{-1} \partial_{r}\left[\left(\psi_{1}+q \phi\right) \omega_{1}^{*}\right]+\text { c.c. }=R e^{-1} \Delta_{0} \omega_{2}, & -\omega_{2}=\Delta_{0} \psi_{2} .
\end{array}
$$

Here $\Delta_{p} \equiv \partial_{r}^{2}+r^{-1} \partial_{r}-n^{2} p^{2} r^{-2}$ and

$$
\alpha \equiv-\frac{1}{r} \partial_{r} \psi_{0}, \quad \beta \equiv \frac{1}{r} \partial_{r} \omega_{0} ;
$$

$\alpha(r)$ is the angular velocity of the axisymmetric flow $\psi_{0}$ and $r \beta(r)$ its vorticity gradient.

The quantities $\omega_{0}, \psi_{0}$ give the basic axisymmetric structure of the vortex; for comparison with previous studies (BL94; Jiménez et al. 1996; BG98) we take a Gaussian vortex

$$
\omega_{0}=(4 \pi)^{-1} \mathrm{e}^{-r^{2} / 4}, \quad \psi_{0}=-\frac{1}{2 \pi}\left[\log r+\int_{r}^{\infty} \mathrm{e}^{-r^{2} / 4} \frac{\mathrm{d} r}{r}\right]
$$


with length scale and circulation of unity. For this vortex

$$
\alpha(r)=\left(2 \pi r^{2}\right)^{-1}\left[1-\exp \left(-r^{2} / 4\right)\right], \quad \beta(r)=-(8 \pi)^{-1} \exp \left(-r^{2} / 4\right)
$$

and as initial conditions we put

$$
\omega=\omega_{0}, \quad \psi=\psi_{0}, \quad \omega_{1}=\omega_{2}=\psi_{1}=\psi_{2}=0 \quad(t=0) .
$$

Note that the non-dimensionalization implicit in (2.8) makes the rotation rate of the vortex quite low. At the centre of the vortex, the angular velocity is $\alpha(0)=1 / 8 \pi$, and the period $16 \pi^{2}$; for this reason the numerical runs below are quite long, and the values of $R e$ taken are large.

The full equations (2.1), (2.2) have a number of inviscid invariants; we shall focus only on the enstrophy $E_{\omega}$ and energy $W$,

$$
E_{\omega}=\frac{1}{2} \int \omega^{2} \mathrm{~d}^{2} \boldsymbol{r}, \quad W=\frac{1}{2} \int \psi \omega \mathrm{d}^{2} \boldsymbol{r} .
$$

The enstrophy $E_{\omega}$ and other moments of the vorticity distribution are conserved for any external flow determined by $q(t)$ because of the material conservation of vorticity. The energy $W$ of the flow is conserved only in the absence of an external flow, $q(t) \equiv 0$ (for a generalization, conserved when $|q(t)|$ and $\mathrm{d}(\arg q) / \mathrm{d} t$ are both constants, see Dritschel 1990, Appendix).

When the expansion (2.5) is substituted in (2.11), the contribution to the enstrophy and energy at $O\left(\varepsilon^{2}\right)$ may be written

$$
\begin{aligned}
e_{\omega} & =\int_{0}^{\infty}\left(\omega_{0} \omega_{2}+\left|\omega_{1}\right|^{2}\right) 2 \pi r \mathrm{~d} r \\
w & =\frac{1}{2} \int_{0}^{\infty}\left(\psi_{0} \omega_{2}+\psi_{2} \omega_{0}+\psi_{1}^{*} \omega_{1}+\psi_{1} \omega_{1}^{*}\right) 2 \pi r \mathrm{~d} r,
\end{aligned}
$$

and these quantities have the same conservation properties as those in (2.11). In these equations we can identify a contribution from the mean parts $\omega_{2}, \psi_{2}, \omega_{0}$ and $\psi_{0}$, and a contribution from the azimuthal part $\omega_{1}$ and $\psi_{1}$. We consider these separately so as to follow the transfer of invariants between mean and azimuthal parts, and use a superscript 'mean' for the axisymmetric contribution and 'azi' for the azimuthal component. For the enstrophy

$$
e_{\omega}^{\text {mean }}=\int_{0}^{\infty} \omega_{0} \omega_{2} 2 \pi r \mathrm{~d} r, \quad e_{\omega}^{\mathrm{azi}}=\int_{0}^{\infty}\left|\omega_{1}\right|^{2} 2 \pi r \mathrm{~d} r
$$

it is the total $e_{\omega}=e_{\omega}^{\text {mean }}+e_{\omega}^{\text {azi }}$ that is conserved inviscidly. Similar remarks apply to the energy $w=w^{\text {mean }}+w^{\text {azi }}$. Note that since $e_{\omega}$ is conserved for any $q(t)$ it follows from the initial condition (2.10) that for $R e=\infty$,

$$
e_{\omega}^{\text {mean }}+e_{\omega}^{\mathrm{azi}}=e_{\omega}=0,
$$

subsequently. However this will not generally be true of the energy $w$.

\subsection{Passive scalar problem}

We now define a passive scalar problem, analogous to the vorticity problem above but much more tractable. We exchange the active scalar, vorticity $\omega$, for the passive scalar $\sigma$ in (2.1) and decouple the stream function in (2.2a), to give

$$
\partial_{t} \sigma=J\left(\psi+\psi_{\mathrm{ext}}, \sigma\right)+P e^{-1} \nabla^{2} \sigma,
$$




$$
\psi \equiv \psi_{0}(r), \quad \nabla^{2} \psi_{\mathrm{ext}}=0 .
$$

Now the flow, given by the total stream function $\psi+\psi_{\text {ext }}$, is totally prescribed and the scalar $\sigma$ obeys a linear equation. We may expand $\sigma$ analogously to (2.5) for vorticity to yield equations similar to (2.6):

$$
\begin{gathered}
\partial_{t} \sigma_{0}=P e^{-1} \Delta_{0} \sigma_{0}, \\
\partial_{t} \sigma_{1}+\mathrm{i} n \alpha \sigma_{1}+\mathrm{i} n \beta q \phi=P e^{-1} \Delta_{1} \sigma_{1}, \\
\partial_{t} \sigma_{2}+\mathrm{i} n r^{-1} \partial_{r}\left(q \phi \sigma_{1}^{*}\right)+\text { c.c. }=P e^{-1} \Delta_{0} \sigma_{2} .
\end{gathered}
$$

Here $P e$ is a Péclet number and

$$
\alpha \equiv-\frac{1}{r} \partial_{r} \psi_{0}, \quad \beta \equiv \frac{1}{r} \partial_{r} \sigma_{0} ;
$$

$\alpha(r)$ is the angular velocity as before, but now $r \beta(r)$ is the basic scalar gradient.

To make closest contact with the vorticity problem, the prescribed axisymmetric flow $\psi_{0}$ is taken to be $(2.8 b)$. Although the initial condition for the scalar may be given independently of the flow field, we choose it to be the same as for vorticity in (2.8a), with

$$
\sigma=\sigma_{0}=(4 \pi)^{-1} \mathrm{e}^{-r^{2} / 4}, \quad \sigma_{1}=\sigma_{2}=0 \quad(t=0) .
$$

In the scalar system we consider only the invariant

$$
E_{\sigma}=\frac{1}{2} \int \sigma^{2} \mathrm{~d}^{2} \boldsymbol{r}
$$

and refer to $E_{\sigma}$ as the scalar variance (although strictly this is $2 E_{\sigma}$ ). The invariance of $E_{\sigma}$ in the absence of diffusion follows from the material conservation of scalar and holds for any $q(t)$. This invariant is analogous to the enstrophy in the vorticity problem, and as there we may define the contribution at order $\varepsilon^{2}$ as $e_{\sigma}$ (cf. 2.12a), and its mean and azimuthal parts, $e_{\sigma}^{\text {mean }}$ and $e_{\sigma}^{\text {azi }}$ (cf. 2.13).

\subsection{Discussion and numerical code}

At leading order in the expansions discussed above we have the basic distributions $\omega_{0}$ and $\sigma_{0}$ of vorticity and scalar, which are subject only to molecular diffusion (if present) by (2.6a), (2.17a) and spread on the long time scale

$$
T_{\text {mean }}=R e \text { or } P e,
$$

respectively. The effect of strain on these basic fields is to generate a purely azimuthal field, $\omega_{1}, \sigma_{1}$, at next order, $O(\varepsilon)$. These azimuthal fields obey the linear equations $(2.6 c, d),(2.17 b)$, forced by the in $\beta q \phi$ term. For both vorticity and scalar the sheardiffuse mechanism operates: once azimuthal vorticity or scalar is driven to small scales it is dissipated on the shear-diffuse time scale,

$$
T_{\mathrm{azi}}=\left(3 R e / n^{2} \alpha^{\prime 2}\right)^{1 / 3} \simeq 20 R e^{1 / 3} \text { or }\left(3 P e / n^{2} \alpha^{2}\right)^{1 / 3} \simeq 20 P e^{1 / 3},
$$

respectively (Lundgren 1982; Moffatt \& Kamkar 1983; BL94). The numerical factor is included here since $\alpha^{\prime}(r)$, although strictly $O(1)$, is small for the Gaussian vortex with $\left|\alpha^{\prime}(r)\right| \lesssim 0.01 . \dagger$

At order $\varepsilon^{2}$ we are primarily interested in the mean fields $\omega_{2}$ and $\sigma_{2}$ which, from

$\dagger$ Note that if $\alpha^{\prime}(r)$ has considerable variation, for example in the case of a point vortex at the origin, the variation of time scale $T_{\text {azi }}$ with radius can lead to anomalous diffusion (Vassilicos 1995). 

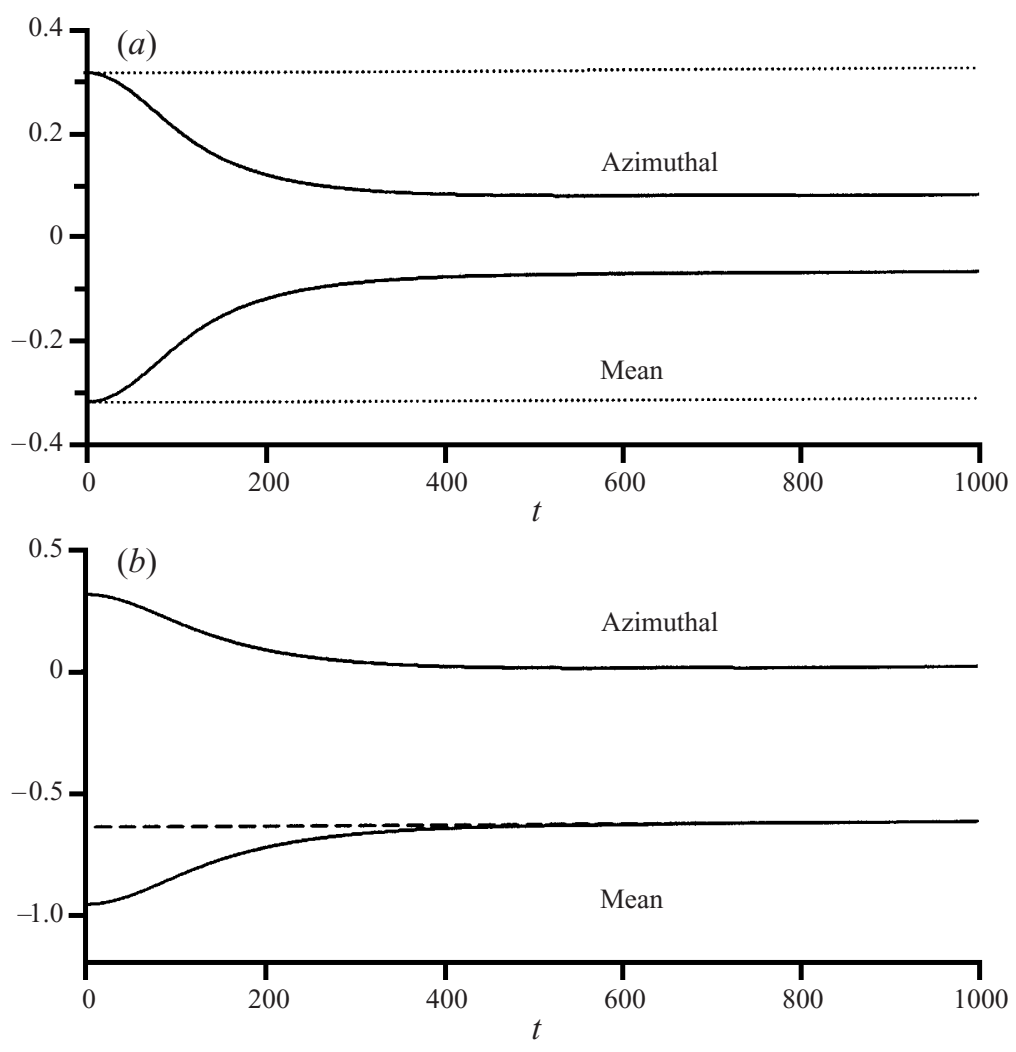

FIGURE 1. (a) Evolution of enstrophy $e_{\omega}$ (solid) and scalar variance $e_{\sigma}$ (dotted) with time; the upper two curves show the azimuthal parts, $e_{\omega}^{\text {azi }}$ and $e_{\sigma}^{\text {azi }}$, the lower two curves the mean parts, $e_{\omega}^{\text {mean }}$ and $e_{\sigma}^{\text {mean }}$. (b) Evolution of the azimuthal energy $w^{\text {azi }}$ (upper solid curve) and the mean energy $w^{\text {mean }}$ (lower solid curve); the total energy $w$ is shown dashed.

(2.6e), $(2.17 c)$, are subject to diffusion on the long time scale (2.21) given above and are forced by the $O(\varepsilon)$ azimuthal fields. We could view the generation of mean field at $O\left(\varepsilon^{2}\right)$ in $\omega_{2}$ as the slow evolution of the $O(1)$ mean field $\omega_{0}$ on a long $O\left(\varepsilon^{-2}\right)$ time scale (and similarly for the scalar), and we take this up in $\S 5$. However in $\S \S 2-4$ we keep $\omega_{0}$ and $\omega_{2}$ distinct.

A numerical code was written to solve the vorticity system (2.6) for $\omega_{0}, \omega_{1}, \omega_{2}$, $\psi_{0}, \psi_{1}$ and $\psi_{2}$, based on that in BG98. Equations (2.6) were rewritten as sixteen real equations of first order in $r$; of these equations four were first order in time. The code used a Keller box method (Keller 1971), as implemented in the NAG library, to step the system in time, with the boundary conditions

$$
\begin{array}{r}
\partial_{r} \omega_{0}=\omega_{1}=\partial_{r} \omega_{2}=\partial_{r} \psi_{0}=\psi_{1}=\partial_{r} \psi_{2}=0 \quad \text { at } r=0, \\
\omega_{0}=\omega_{1}=\omega_{2}=0, \quad \psi_{0}=-(2 \pi)^{-1} \log r, \quad r \partial_{r} \psi_{1}+n \psi_{1}=0, \quad \psi_{2}=0 \quad \text { at } r=r_{\max } .
\end{array}
$$

For the passive scalar a similar code was written to solve (2.17) for $\sigma_{0}, \sigma_{1}$ and $\sigma_{2}$ with

$$
\partial_{r} \sigma_{0}=\sigma_{1}=\partial_{r} \sigma_{2}=0 \quad \text { at } r=0, \quad \sigma_{0}=\sigma_{1}=\sigma_{2}=0 \quad \text { at } r=r_{\max } .
$$


Typical parameter values for the computations described in $\S \S 3,4$ are

$$
r_{\max }=10, \quad N=1001, \quad R e=P e=10^{8},
$$

$N$ being the number of spatial grid points, spaced evenly between $r=0$ and $r=r_{\max }$. The codes were tested by verifying the conservation of vorticity and scalar invariants at high $R e$ and $P e$. In particular the conservation of energy $w$ (as seen for the dashed line in figure $1 b$ ) is a good test of the vorticity code, as it verifies the calculation of all the various components of the stream function.

In $\S 3,4$ our interest is in the strictly non-diffusive behaviour of vorticity and a passive scalar, but we have to introduce diffusion in the code for numerical reasons. However for the parameters used, the time scales for the viscous decay of mean and azimuthal fields are

$$
T_{\text {mean }}=10^{8}, \quad T_{\text {azi }} \simeq 10^{4} .
$$

These are both much longer than the runs presented, and so the results given in these sections do show the behaviour of the non-diffusive case, $R e=P e=\infty$. Irrespective of diffusion, there is also the issue of the validity of the expansion (2.5) in powers of $\varepsilon$, in the limit of long time $t$. It appears that the series remains uniform for arbitrarily large $t$ for $\varepsilon \ll 1$ in $\S 3$ and for $\varepsilon t_{\max } \ll 1$ in $\S 4$. The reason is that once the forcing $q(t)$ is turned off, the azimuthal fields relax to axisymmetry, the feedback to the mean fields $\omega_{2}, \sigma_{2}$ ceases, and the mean fields remain bounded subsequently, ensuring that the series does not become disordered.

\section{Response to an impulsive external strain}

In this section we study the response of a vortex to an impulsive external strain. We take

$$
q(t)=2 t_{\max }^{-1} \sin ^{2}\left(\pi t / t_{\max }\right) \quad\left(0<t<t_{\max }\right),
$$

and $q(t)=0$ otherwise. This is a continuous input, for numerical reasons, but provided $t_{\max }$ is sufficiently small, it is a good approximation to a delta function $q(t)=\delta(t)$ from the point of view of the linear equations for $\omega_{1}$ and $\sigma_{1}$. For small $t_{\max }$ it is equivalent to imposing the initial condition,

$$
\omega_{1}\left(r, 0^{+}\right)=-\mathrm{i} n \beta \phi, \quad \sigma_{1}\left(r, 0^{+}\right)=-\mathrm{i} n \beta \phi,
$$

on these fields. The subsequent linear evolution of vorticity, which gives a Green's function, was studied for $1 \ll R e<\infty$ in BL94 and for $R e=\infty$ in BG98. We take $t_{\max }=1$, which is short compared with all other time scales in the problem.

\subsection{Transfer of enstrophy and scalar variance}

The vorticity field is evolved under the near-impulsive input (3.1) with $t_{\max }=1$. In figure $1(a)$ we show the evolution of the azimuthal enstrophy $e_{\omega}^{\text {azi }}$ (upper solid curve) and the mean enstrophy $e_{\omega}^{\text {mean }}$ (lower solid curve) as functions of time. The dotted lines show the corresponding mean and azimuthal scalar variances under the same input. At $t=0$ all these quantities are zero by (2.10), (2.19), but during the input for $0 \leqslant t \leqslant 1$ there is a transfer of enstrophy from mean to azimuthal component. At $t=1$,

$$
e_{\omega}^{\mathrm{azi}}=-e_{\omega}^{\text {mean }}=e_{\sigma}^{\mathrm{azi}}=-e_{\sigma}^{\text {mean }} \simeq 1 / \pi \simeq 0.318
$$

from (3.2); for short times (too short to be visible in the figure) the scalar and vorticity behave identically. 
However for $t>1$ the enstrophy curves (solid) and scalar variance curves (dotted) diverge. The scalar quantities remain constant for $t>1$. This is no great surprise: when $q(t)=0$ the flow possesses only circular streamlines, and from $(2.17 b)$ with $P e=\infty$

$$
\partial_{t}\left|\sigma_{1}\right|^{2}=0, \quad \sigma_{1}(r, t)=-\mathrm{i} n \beta \phi \mathrm{e}^{-\mathrm{i} n \alpha(r) t} .
$$

As a consequence $e_{\sigma}^{\text {azi }}$ and so $e_{\sigma}^{\text {mean }}$ are individually conserved for $q(t)=0$, as seen. No similar deductions can be made for $e_{\omega}^{\text {azi }}$ and $e_{\omega}^{\text {mean }}$ as the azimuthal vorticity $\omega_{1}$ generates a stream function $\psi_{1}$ which causes motion in and out of the vortex, allowing a flow of enstrophy between mean and azimuthal components. This is evident in figure $1(a)$ where for the vorticity there is a 'rebound' phenomenon, a transfer of enstrophy from the azimuthal component back to the mean. By the end of the run $e_{\omega}^{\text {azi }}=-e_{\omega}^{\text {mean }} \simeq 0.075$ and so the mean and azimuthal enstrophies are reduced by about a quarter. This suppression of $e_{\omega}^{\text {azi }}$ was mentioned in BG98, and arises purely through the linear evolution equations $(2.6 c, d)$.

Although dissipation is negligible during the numerical simulation in figure 1(a), the above results have implications for the later dissipation of enstrophy and scalar variance, because of the differing mechanisms for mean and azimuthal components. From the results above the enstrophy destroyed by the shear-diffuse mechanism will be $e_{\omega}^{\text {azi }} \simeq 0.075$ at a time $t=O\left(R e^{1 / 3}\right)$. The scalar variance destroyed will be much greater, namely $e_{\omega}^{\mathrm{azi}} \simeq 0.318$ at a time $t=O\left(P e^{1 / 3}\right)$. The mean fields will only be influenced by diffusion on the much longer $O(R e)$ and $O(P e)$ time scales.

Even in the strictly non-dissipative situation $R e=P e=\infty$, both azimuthal fields are driven to small scales by differential rotation and effectively lost to the system on the $O(1)$ turnover time scale of the vortex; however again less enstrophy is lost in this way than passive scalar variance. In this sense the vorticity distribution is more robust than the analogous passive scalar. Even more robust is the Kida (1981) vortex: under a weak impulsive strain, an initially circular vortex patch would be distorted into an ellipse, which would rotate as a solid body thereafter, and there would be no enhanced dissipation due to spiral wind-up.

To attempt to throw some light on the suppression of azimuthal enstrophy $e_{\omega}^{\text {azi }}$ let us take $q(t)=0$ and $R e=\infty$ and consider

$$
\partial_{t}\left|\omega_{1}\right|^{2}=\operatorname{in} \beta\left(\omega_{1} \psi_{1}^{*}-\omega_{1}^{*} \psi_{1}\right)
$$

from $(2.6 c)$. For vorticity we lack an explicit solution analogous to $(3.4 b)$ and so the behaviour for the most interesting times $t=O(1)$ remains beyond our reach. We can however study the suppression of azimuthal vorticity for large time $t$, when the vorticity tends to axisymmetry and a long-time asymptotic solution is available. As $t \rightarrow \infty$ the solution to $(2.6 c, d)$ is given by

$$
\omega_{1}=\omega_{\mathrm{L}}+\omega_{\mathrm{H}}, \quad \psi_{1}=\psi_{\mathrm{L}}+\psi_{\mathrm{H}},
$$

where

$$
\omega_{\mathrm{L}}=\left(X_{0}+t^{-1} X_{1}+O\left(t^{-2}\right)\right) \mathrm{e}^{-\mathrm{in} \alpha(r) t}, \quad \psi_{\mathrm{L}}=\left(t^{-2} Y_{0}+t^{-3} Y_{1}+O\left(t^{-4}\right)\right) \mathrm{e}^{-\mathrm{i} n \alpha(r) t},
$$

with

$$
\begin{gathered}
X_{0}=g(r), \quad X_{1}=\mathrm{i} n \beta Y_{0}, \\
Y_{0}=\frac{g(r)}{n^{2} \alpha^{\prime 2}}, \quad Y_{1}=\frac{\mathrm{i} n}{n^{2} \alpha^{\prime 2}}\left[\left(\beta-\alpha^{\prime \prime}-\frac{\alpha^{\prime}}{r}\right) Y_{0}-2 \alpha^{\prime} Y_{0}^{\prime}\right] .
\end{gathered}
$$


This is Lundgren's (1982, appendix A) spiral solution taken to second order (see $\S 5$ of BG98). The series involves a single complex function $g(r)$ : although this is not known analytically, as it only emerges after evolution through times $t=O(1)$, in principle it is determined by the initial conditions, and can be found numerically.

The second part of the solution $\omega_{\mathrm{H}}, \psi_{\mathrm{H}}$ was referred to as the Helmholtz solution in BG98. This solution decays according to

$$
\omega_{\mathrm{H}}, \psi_{\mathrm{H}}=O\left(t^{-\lambda}\right), \quad \lambda=1+\sqrt{n^{2}+8}
$$

(cf. Briggs et al. 1970), and because of this the Lundgren solution (3.7) always gives the dominant contribution to the quantities discussed in this paper. We therefore do not consider the Helmholtz solution further, although its presence has implications for the far field of the vortex and the behaviour near the origin.

When these expansions are substituted into (3.5) we obtain

$$
\partial_{t}\left|\omega_{1}\right|^{2}=-2 n^{2} t^{-3} \beta r^{-1} \partial_{r}\left(r \alpha^{\prime}\left|Y_{0}\right|^{2}\right)+O\left(t^{-5}\right) ;
$$

the right-hand side does not arise at leading order from (3.7) but from the next-order cross-terms $X_{0} Y_{1}^{*}, X_{1} Y_{0}^{*}$ and their complex conjugates. Integrating over the vortex in $(2.13 b)$ and using integration by parts gives

$$
\partial_{t} e_{\omega}^{\mathrm{azi}}=2 n^{2} t^{-3} \int_{0}^{\infty} \alpha^{\prime} \beta^{\prime}\left|Y_{0}\right|^{2} 2 \pi r \mathrm{~d} r+O\left(t^{-5}\right) .
$$

For the Gaussian vortex (2.8), $\alpha^{\prime} \leqslant 0$ and $\beta^{\prime} \geqslant 0$ from (2.9), and so the integral is negative definite. Thus as we observe numerically, at large times $t$ there is decay of the azimuthal component $e_{\omega}^{\text {azi }}$ of the enstrophy, and a balancing generation of the mean $e_{\omega}^{\text {mean }}$.

This raises the question of how our (somewhat arbitrary) choice of the Gaussian vortex as initial condition influences the numerical results we observe, in particular the rebound of enstrophy from mean to azimuthal components. Investigation of this issue remains a topic for future research; however we note that from $(2.6 b),(2.7)$ it follows that $\beta^{\prime}=r^{-3} \partial_{r}\left(r^{3} \alpha^{\prime}\right)$ and then integrating by parts shows that

$$
\int_{0}^{\infty} \alpha^{\prime} \beta^{\prime} r^{3} \mathrm{~d} r=-\int_{0}^{\infty} \beta^{2} r^{3} \mathrm{~d} r<0 .
$$

Thus the combination $\alpha^{\prime} \beta^{\prime}$ is either negative definite or indefinite in sign, but cannot be positive definite. One can construct examples of vortices for which it is indefinite, but then the sign of the weighted integral (3.11) giving the long-time behaviour of azimuthal enstrophy is not known without further information about the elusive function $g(r)$ in (3.8).

Figure $1(b)$ shows the evolution of the energy $(2.12 b)$ for the case of vorticity. The upper solid curve shows the azimuthal energy $w^{\text {azi }}$ and the lower solid curve $w^{\text {mean }}$. The dashed line is the total energy $w$; this decreases to $w \simeq-0.64$ while the forcing acts in $0<t<1$ and subsequently is constant. As $t \rightarrow \infty$, the azimuthal energy is

$$
w^{\mathrm{azi}}=t^{-2} \int\left|\frac{g(r)}{n \alpha^{\prime}}\right|^{2} 2 \pi r \mathrm{~d} r+O\left(t^{-3}\right),
$$

from (3.7), (3.8), and tends to zero. This decay is a consequence of the relaxation of the vortex to axisymmetry and the fact that very fine-scaled distributions of vorticity carry negligible energy. At the same time the mean energy increases, and asymptotically the net loss of energy is only evident in this mean distribution. 

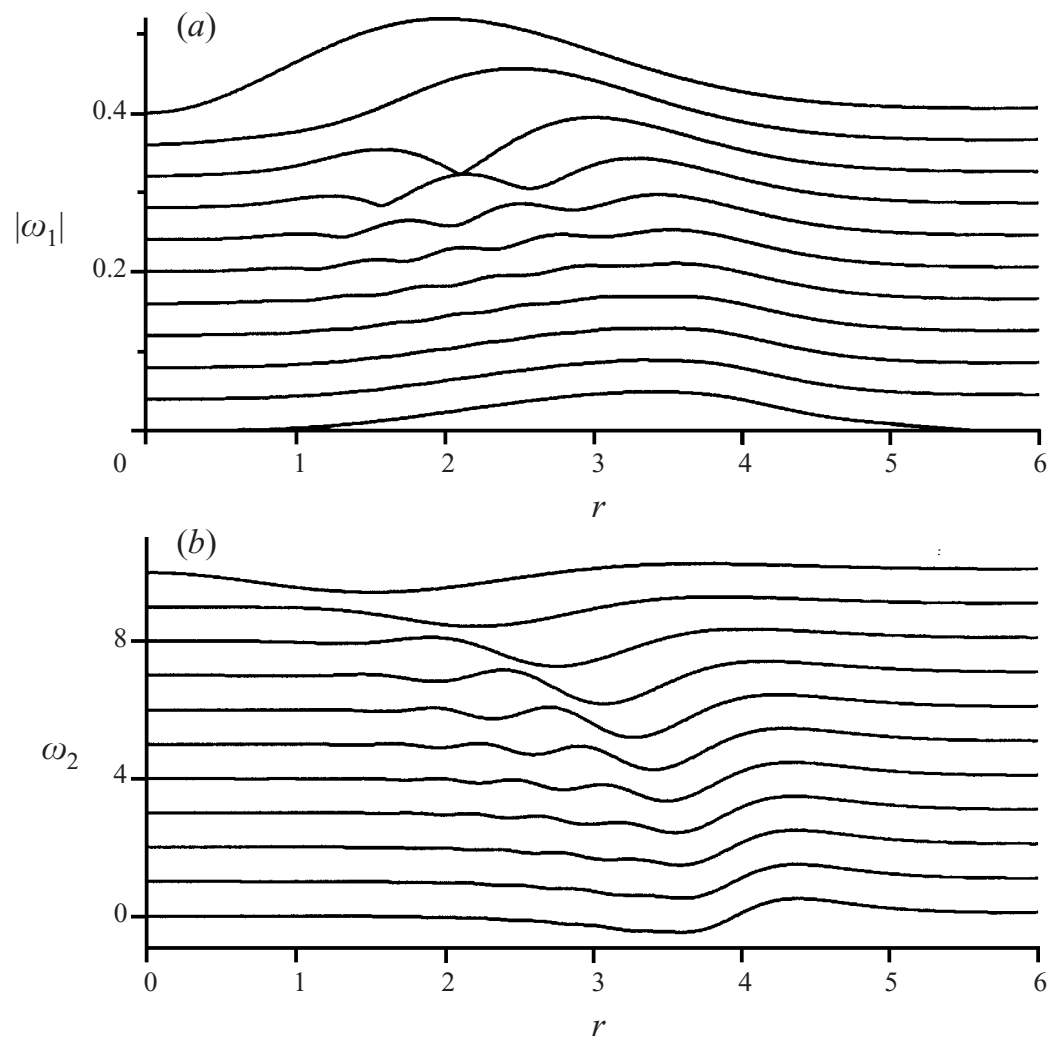

FiguRE 2. (a) Evolution of $\left|\omega_{1}\right|$ as a function of $r$ for $t=1,100, \ldots, 1000$, reading downwards; the curves have been separated by adding constants. The curve for $t=1$ also gives $\left|\sigma_{1}\right|$ for $t \geqslant 1$. (b) Evolution of $\omega_{2}$ as a function of $r$ for the same set of times. The curve for $t=1$ also gives $\sigma_{2}$ for $t \geqslant 1$.

\subsection{Structure in physical space}

Let us consider the structure of scalar and vorticity fields in more detail. At large times the complex function $\omega_{1}$ giving the azimuthal vorticity field oscillates rapidly in radius; we plot its envelope $\left|\omega_{1}\right|$ against $r$ in figure $2(a)$ which shows a sequence for $t=1,100,200, \ldots, 1000$, reading downwards. (The curves are separated by adding constants.) Note the presence of kinks at moderate times $t \simeq 200-400$, discussed further below. We also observe that as time increases there is an overall suppression of the level of the azimuthal vorticity, which confirms the results for enstrophy above. The suppression is particularly noticeable near the origin, and the maximum value of $\left|\omega_{1}\right|$ moves outwards with time: at $t=1$ the maximum is $\left|\omega_{1}\right| \simeq 0.12$ at $r \simeq 2$, whilst at $t=1000$ the maximum is 0.047 at $r \simeq 3.4$.

This outwards motion of vorticity fluctuations is also observed in Montgomery \& Kallenbach (1997), who ascribe it to vortex Rossby waves with a group velocity that has an outwards radial component. Another view of this process is seen by integrating (3.5) to yield

$$
\partial_{t} \int_{0}^{\infty} \beta^{-1}\left|\omega_{1}\right|^{2} r \mathrm{~d} r=\int_{0}^{\infty} \mathrm{in}\left(\omega_{1} \psi_{1}^{*}-\omega_{1}^{*} \psi_{1}\right) r \mathrm{~d} r=0,
$$

using $\omega_{1}=-\Delta_{1} \psi_{1}$ and integration by parts. Within our perturbative scheme, this 
(a)

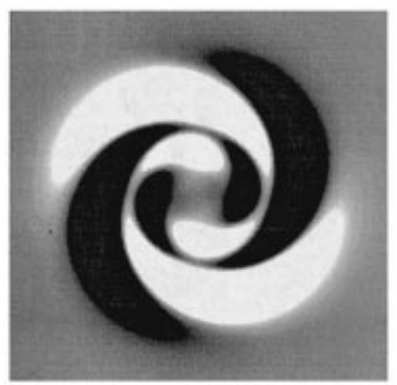

$(d)$

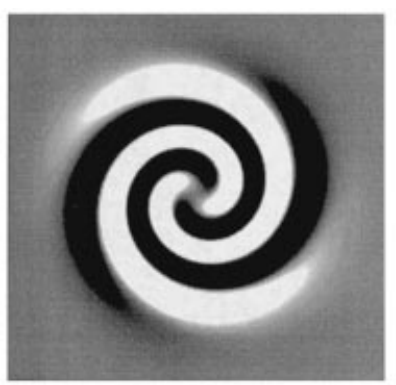

A. P. Bassom and A. D. Gilbert

(b)

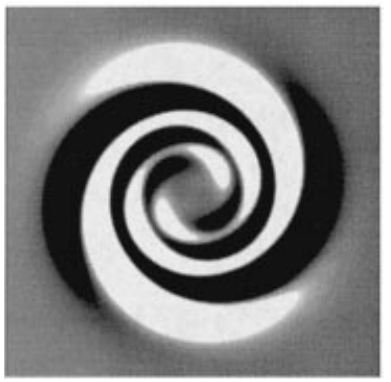

$(e)$

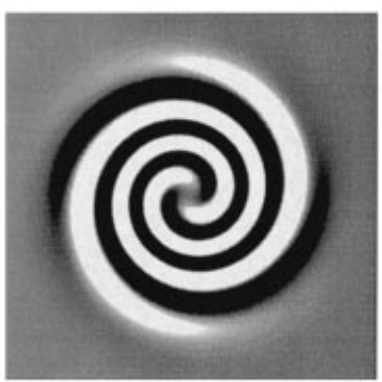

(c)

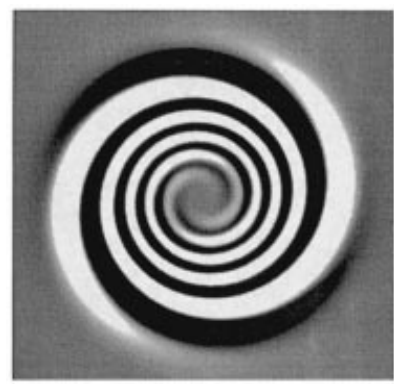

$(f)$

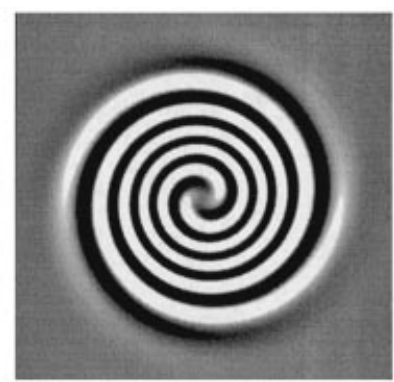

Figure 3. $(a, b, c)$ Vorticity field $\hat{\omega}_{1}(r, \theta)$ and $(d, e, f)$ scalar field $\hat{\sigma}_{1}(r, \theta)$, for $-6 \leqslant x, y \leqslant 6$. In $(a, d)$ $t=200$, in $(b, e) t=300$ and in $(c, f) t=500$, for the impulsive input of $\S 3$. The scale is white where $10 \hat{\omega}_{1}>\max \left|\hat{\omega}_{1}\right|$ and black where $10 \hat{\omega}_{1}<-\max \left|\hat{\omega}_{1}\right|$, values close to zero being grey.

weighted integral of $\left|\omega_{1}\right|^{2}$ is conserved for all $t$ in the absence of forcing, and in the Gaussian vortex $\beta$ is of one sign. If we therefore consider large finite times $t$, then we know from (3.14) that the integral of $\beta^{-1}\left|\omega_{1}\right|^{2}$ is constant while the integral of $\left|\omega_{1}\right|^{2}$ (which is $2 e_{\omega}^{\mathrm{azi}}$ ) is decaying from (3.11). For these two behaviours to hold simultaneously there must be a general tendency for $\left|\omega_{1}\right|^{2}$ to increase in regions of low $|\beta|$ and decrease in regions of high $|\beta|$. Since $|\beta|$ is largest in the centre of the vortex, this implies a shift of $\left|\omega_{1}\right|^{2}$ from the centre to the periphery, as observed.

Figure $2(b)$ shows the corresponding time sequence for the mean vorticity perturbation $\omega_{2}$. Once more there is a movement outwards to greater radii, as seen by Montgomery \& Kallenbach (1997). For large times the net effect of the perturbation is only to modify the mean flow at quite large radii, the centre remaining relatively unperturbed. The behaviour of the passive scalar is simpler than vorticity because for $t>1,\left|\sigma_{1}\right|$ and $\sigma_{2}$ are constant as functions of radii. In fact $\left|\sigma_{1}\right|$ and $\sigma_{2}$ for $t \geqslant 1$ are practically indistinguishable from $\left|\omega_{1}\right|$ and $\omega_{2}$ for $t=1$, shown as the top curve of figures $2(a)$ and $2(b)$ respectively. With

Finally we reconstruct the azimuthal vorticity and scalar fields as grey-scale plots.

$$
\hat{\omega}_{1}(r, \theta, t)=\omega_{1}(r, t) \mathrm{e}^{\mathrm{i} n \theta}+\text { c.c., } \quad \hat{\sigma}_{1}(r, \theta, t)=\sigma_{1}(r, t) \mathrm{e}^{\mathrm{i} n \theta}+\text { c.c. },
$$

figures $3(a)-3(c)$ show $\hat{\omega}_{1}$ for $t=200,300$ and 500, respectively. Figures $3(d)-3(f)$ show the same sequence for $\hat{\sigma}_{1}$. The spiral arms generated by differential rotation show more internal structure for vorticity than for a passive scalar (cf. Montgomery $\&$ Kallenbach 1997). In figure $3(a)$ for $\hat{\omega}_{1}$, there are essentially two distorted vortices in each arm; these appear to result from a secondary instability reminiscent of 
Kelvin-Helmholtz roll-up. There are also radii at which there is very little vorticity, and these correspond to the downward spikes of low $\left|\omega_{1}\right|$ seen in figure 2(a) for $t=200-400$. However by the latest time $t=500$ (figure $3 c, f$ ) the spiral arms in $\omega$ have become uniform under the influence of differential rotation, which has the effect of suppressing Kelvin-Helmholtz instabilities (Dritschel 1989b; Dritschel et al. 1991; Kevlahan \& Farge 1997). The suppression of vorticity in the centre of the vortex, relative to the passive scalar, can be seen but is not prominent because the grey-scale coding, detailed in the caption, is designed rather to emphasize structure in the spiral arms.

\section{Response to a step input}

Above we studied the response of the vorticity and scalar systems to an impulsive external strain $q(t)=\delta(t)$, typical of a short correlation-time input. A contrasting situation, typical of a long correlation time, is to switch on a constant strain field $q=1$ at $t=0$ and to observe how the vortex relaxes to its new environment. This constant strain is given by

$$
\psi_{\mathrm{ext}}=2 \varepsilon r^{2} \cos 2 \theta=2 \varepsilon\left(x^{2}-y^{2}\right)
$$

from $(2.4 a, b)$ with $n=2$. The Gaussian vortex will relax to take the form of a strained vortex, plus fluctuations which will be subject to differential rotation and ultimately dissipation; again our interest is to compare the behaviour of vorticity and passive scalar $(\$ \$ 4.1,4.2)$. When the external strain is subsequently switched off, the vortex relaxes to axisymmetry, and we determine how much enstrophy is destroyed in the process $(\S 4.3)$. In this section we take

$$
q(t)=1 \quad\left(0<t<t_{\max }\right), \quad q(t)=0 \quad\left(t<0, t>t_{\max }\right),
$$

with $t_{\max }=1000$. For numerical reasons the jump at $t=t_{\max }$ is in fact smoothed by putting $2 q(t)=\left(1-\tanh \left(t-t_{\max }\right)\right)$.

\subsection{Relaxation to the external strain field, $t<t_{\max }$}

For the step-function input, figure $4(a, b)$ parallels figure $1(a, b)$. Figure $4(a)$ shows the azimuthal (upper) and mean (lower) enstrophy (solid) and scalar variance (dotted) as functions of time. Figure $4(b)$ shows the evolution of energy for the vorticity problem. The time $t_{\max }=1000$ is marked with a vertical dashed line. From figure 4(a), for $t<t_{\max }$ we observe initial damped oscillations as enstrophy and scalar variance are transferred between mean and azimuthal fields. More enstrophy is transferred from the mean into the azimuthal component than occurs for the scalar variance; at $t=1000, e_{\omega}^{\mathrm{azi}}=-e_{\omega}^{\mathrm{mean}} \simeq 690$ and $e_{\sigma}^{\mathrm{azi}}=-e_{\sigma}^{\mathrm{mean}} \simeq 440$.

This strong response of the vorticity field arises through the positive feedback mechanism depicted in figure 5. Kinematically, the sum of an axisymmetric stream function and the strain field $(a)$ gives elliptical stream lines $(b)$. A passive scalar will relax to a distribution constant on these stream lines, corresponding to positive and negative $\sigma_{1}$ as indicated in $(c)$. When the scalar is instead vorticity this distribution $\omega_{1}$ generates a flow which tends to reinforce the original external strain $(d)$. The result is that the vorticity equilibrates at a greater amplitude than a passive scalar. This feedback mechanism is presumably responsible for the initial transient oscillations of enstrophy seen in figure 4(a), which are of large amplitude. These results are in accord with Ohkitani (1991), who found greater fluctuations in enstrophy transfer (by comparison with a passive scalar) in two-dimensional turbulence. Note however 

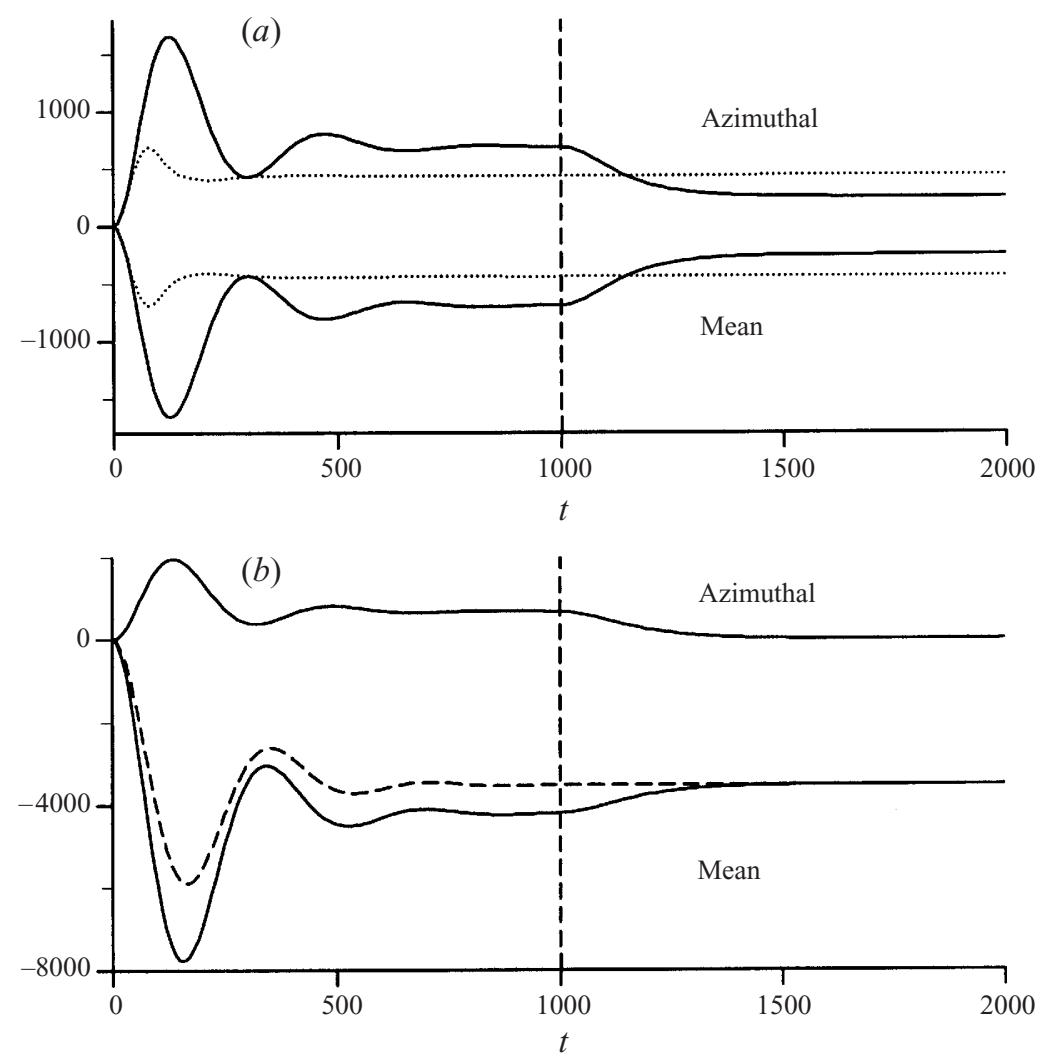

FIGURE 4. (a) Evolution of enstrophies $e_{\omega}$ (solid) and $e_{\sigma}$ (dotted) with time for the step input of $\S 4$; the upper two curves show the azimuthal parts, $e_{\omega}^{\mathrm{azi}}$ and $e_{\sigma}^{\mathrm{azi}}$, the lower two curves the mean parts, $e_{\omega}^{\text {mean }}$ and $e_{\sigma}^{\text {mean }}$. (b) Evolution of the azimuthal energy $w^{\text {azi }}$ (upper solid curve) and the mean energy $w^{\text {mean }}$ (lower solid curve); the total energy is shown dashed. In each part the vertical dashed line marks the time $t=t_{\max }$ when the external strain is switched off.

that for three-dimensional vortices strained along their axes, the feedback is such as to reduce the external strain (Andreotti, Douady \& Couder 1996).

The damped oscillations seen may be contrasted with Kida's (1981) elliptical vortex model: a circular vortex patch placed in a steady strain field will oscillate indefinitely between elliptical and circular states (this is the case $s=1$ on figure 1 of Kida 1981). There is no irreversible transfer of enstrophy to small scales and so it is not possible for the vortex to relax to the stable steady-state solution of an elliptical vortex with principal axes at $\pi / 4$ to the strain axes (Moore \& Saffman 1971).

The vorticity $\omega_{1}$ and scalar $\sigma_{1}$ are shown in figure $6(a, b)$ as functions of radius, at $t=t_{\max }$; the real parts are shown solid, imaginary parts dotted. Figure $7(a, b)$ shows the corresponding two-dimensional distributions $\hat{\omega}_{1}$ and $\hat{\sigma}_{1}$ (see (3.15)). In each case the fields clearly take the form of a dipolar distribution, on which are superposed oscillations. This behaviour may easily be confirmed for the passive scalar by solving (2.17b) for $q=1, P e=\infty$ and zero initial conditions, giving

$$
\sigma_{1}=\left(1-\mathrm{e}^{-\mathrm{i} n \alpha(r) t}\right) \Sigma_{1}(r), \quad \Sigma_{1}(r) \equiv-\phi \beta / \alpha .
$$

Here $\Sigma_{1}(r)$ satisfies the steady version of $(2.17 b)$ while the exponential term takes account of the zero initial conditions. The solution for $\sigma_{1}$ (solid and dotted) in figure 
(a)

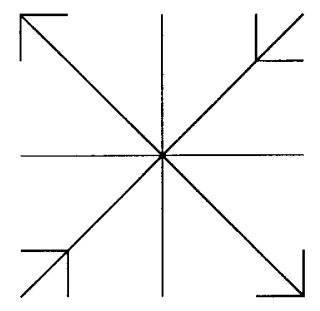

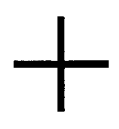

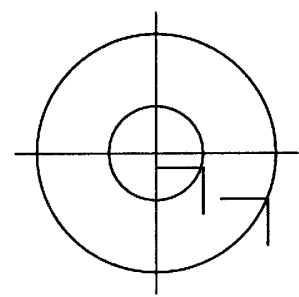

(b)

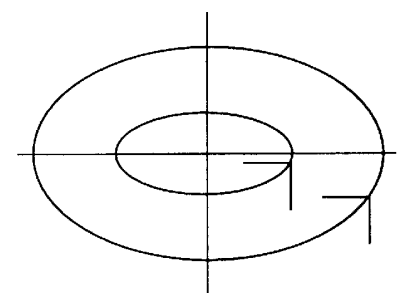

(c)

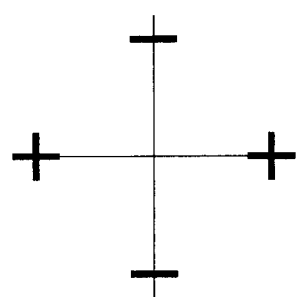

(d)

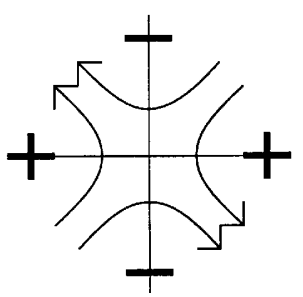

FiguRE 5. (a) The sum of an axisymmetric stream function and the strain field gives $(b)$ elliptical stream lines. (c) Resultant distribution for a passive scalar. (d) Resultant secondary flow for vorticity.

6(b) consists of the steady component $\Sigma_{1}$ (shown dashed), superposed by oscillations that vary more rapidly with radius as time increases.

Although plainly $\sigma_{1}$ does not tend to $\Sigma_{1}$ pointwise, it does in a weak sense quantified in BG98. There we defined an inner product by $(F, G)=\int F^{*} G r \mathrm{~d} r \mathrm{~d} \theta$, the integral being taken over all space. Given a smooth test function $F(r, \theta)$ a spatial average of $\sigma(r, \theta, t)$ may be constructed by forming $(F, \sigma)$. Here we take the test function $F(r, \theta)=f(r) \mathrm{e}^{\mathrm{i} n \theta}$ and

$$
\left(f \mathrm{e}^{\mathrm{i} n \theta},\left(\sigma_{1}-\Sigma_{1}\right) \mathrm{e}^{\mathrm{i} n \theta}\right)=O\left(t^{-n-1}\right)
$$

in general (BG98). Thus while $\sigma_{1}$ does not tend to $\Sigma_{1}$ pointwise, spatial averages do, and in this sense the scalar field $\sigma_{1}$ relaxes to the solution $\Sigma_{1}$ of the steady equation (2.17b).

Similar arguments apply to the vorticity, although explicit solutions are not available; the vorticity $\omega_{1}$ appears to relax to a solution $\Omega_{1}$ of the steady governing equations $(2.6 c, d)$, which satisfies

$$
\Delta_{1} \Psi_{1}-(\beta / \alpha) \Psi_{1}=\beta \phi / \alpha, \quad-\Omega_{1}=\Delta_{1} \Psi_{1} .
$$

When the functions $\alpha$ and $\beta$ refer to the Gaussian vortex (2.8), as here, the solution to this equation has been studied by Moffatt et al. (1994), and gives the structure of a viscously controlled vortex in an external strain flow. In our case the vortex is not viscously controlled and this correspondence is in fact a coincidence arising from our convenient but arbitrary choice of the Gaussian vortex as initial condition. As for the scalar, the vorticity field $\omega_{1}$ does not tend to $\Omega_{1}$ pointwise, but instead spatial averages converge with

$$
\left(f \mathrm{e}^{\mathrm{i} n \theta},\left(\omega_{1}-\Omega_{1}\right) \mathrm{e}^{\mathrm{i} n \theta}\right)=O\left(t^{-\lambda}\right),
$$



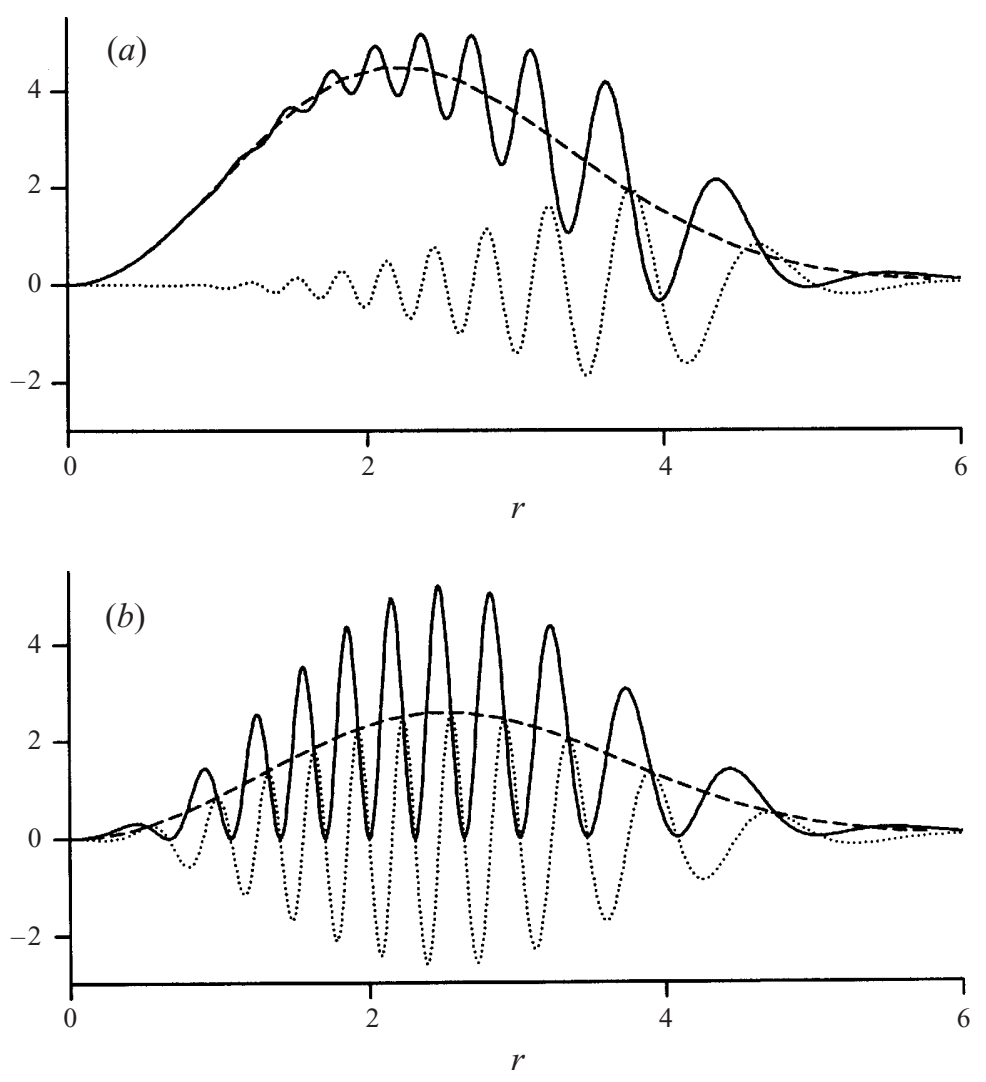

FiguRE 6. (a) $\omega_{1}$ shown as a function of $r$ for $t=t_{\max }$, with real part shown solid and imaginary part dotted. The dashed curve gives $\Omega_{1}$ (see (4.5)). (b) $\sigma_{1}$ shown as a function of $r$ for $t=t_{\max }$, with real part shown solid and imaginary part dotted. The dashed curve gives $\Sigma_{1}$ (see (4.3)).

where $\lambda$ was defined in (3.9) above (BG98). At time $t=t_{\max }$ figure 6(a) shows the vorticity $\omega_{1}$ (solid, dotted) which takes the form of $\Omega_{1}$ (dashed) with superposed oscillations, which are suppressed near the origin. The vorticity distribution is generally more coherent than that of the passive scalar in figure $6(b)$, and this is confirmed in the grey-scale pictures for vorticity and scalar, figures $7(a)$ and $7(b)$ respectively.

\subsection{Feedback to the mean vorticity and scalar}

When the strain field is turned on at $t=0$, then together with the generation of the azimuthal fields $\sigma_{1}$ and $\omega_{1}$ there is also a feedback to the axisymmetric mean fields at $O\left(\varepsilon^{2}\right)$. For the passive scalar we can calculate this explicitly from (2.17c) with $P e=\infty$ :

$$
\sigma_{2}=r^{-1} \partial_{r}\left[2 \phi^{2} \beta \alpha^{-2}(1-\cos n \alpha(r) t)\right] .
$$

Again at large time $t$ the term $\cos n \alpha t$ oscillates rapidly as a function of radius; if it is ignored we obtain a steady-state distribution,

$$
\Sigma_{2}=r^{-1} \partial_{r}\left(2 \phi^{2} \beta \alpha^{-2}\right),
$$

which can be written in the suggestive form (by substituting $\beta$ from (2.18b)),

$$
\Sigma_{2}=r^{-1} \partial_{r}\left(r \kappa(r) \partial_{r} \sigma_{0}\right), \quad \kappa(r)=2 \phi^{2} / r^{2} \alpha^{2} .
$$


(a)

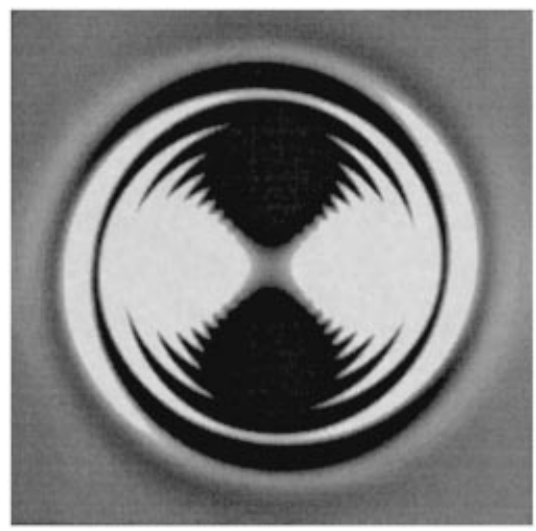

(b)

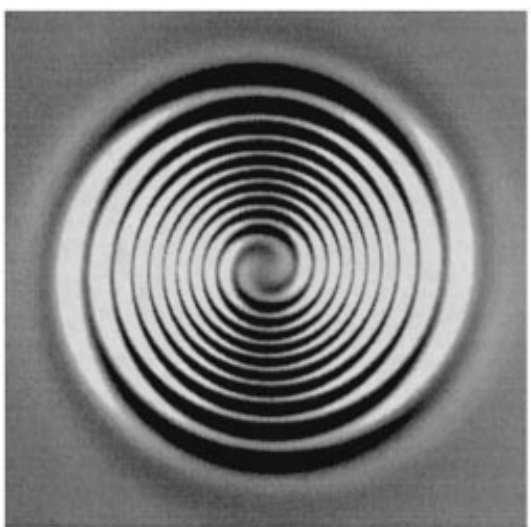

FIGURE 7. ( $a$ ) Vorticity field $\hat{\omega}_{1}(r, \theta)$ and $(b)$ scalar field $\hat{\sigma}_{1}(r, \theta)$ for $t=t_{\max }$ with the step-function input of $\S 4$. The scales are as in figure 3 .

The modification to the mean profile takes the form of a diffusive spreading, where $\kappa(r)$ is a radius-dependent effective diffusivity.

The relaxation of $\sigma_{2}$ to $\Sigma_{2}$ is however not pointwise. In fact the maximum difference between the two diverges as $O(t)$ for large $t$ (and so the perturbation series in $\varepsilon$ becomes non-uniform for $\varepsilon t=O(1)$ ). However in the weak sense outlined above $\sigma_{2}$ does relax to $\Sigma_{2}$ : this follows by taking $F(r, \theta)=f(r)$ as a smooth axisymmetric test function and considering

$$
\left(f, \sigma_{2}-\Sigma_{2}\right)=\left(f,-r^{-1} \partial_{r}\left(2 \phi^{2} \beta \alpha^{-2} \cos n \alpha t\right)\right)=\left(r^{-1} \partial_{r} f, 2 \phi^{2} \beta \alpha^{-2} \cos n \alpha t\right),
$$

using integration by parts. At the origin $\phi^{2} \beta \alpha^{-2}=O\left(r^{2 n}\right)$ and so using the methods in BG98 it may be shown that

$$
\left(f, \sigma_{2}-\Sigma_{2}\right)=O\left(t^{-n-1}\right),
$$

establishing the desired weak convergence.

To summarize, at the level of $\sigma_{2}$ there is a spreading of the scalar distribution, which can be considered one step of a diffusive process yielding $\Sigma_{2}$. Superposed on this are oscillations (which grow secularly with time and are a sign that perturbation theory is breaking down), but these oscillations are tending to zero in a weak sense, and so are unimportant. This suggests that a time-dependent, random strain field will lead to a continuous diffusive spreading of the scalar distribution, as we will confirm in $\S 5$ below. We can say very little about the corresponding modification $\omega_{2}$ to the mean vorticity distribution, except that we expect this to relax in a weak sense to a profile $\Omega_{2}$. However in view of the non-local nature of the vorticity problem it is unlikely that $\Omega_{2}$ can be expressed analogously to $\Sigma_{2}$ above in terms of a local effective diffusivity.

\subsection{Relaxation to axisymmetry, $t>t_{\max }$}

For $t>t_{\max }=1000$, the external strain is switched off, $q(t)=0$, and the vortex relaxes to an axisymmetric configuration once more. Returning to figure $4(a)$, we observe that for enstrophy (solid) there is a transfer from the azimuthal component to the mean after $t=t_{\max }$ (marked by the vertical dashed line), which does not occur for the scalar variance (dotted). At the end of the run the azimuthal enstrophy 
$e_{\omega}^{\text {azi }} \simeq 250$ is lower than the azimuthal scalar variance $e_{\sigma}^{\text {azi }} \simeq 440$. As in $\S 3$ this rebound phenomenon means that less enstrophy than scalar variance is lost to fine scales and so to dissipation by the shear-diffuse mechanism, if dissipation is present.

While the rebound of the enstrophy from azimuthal to mean component seen in figure $4(a)$ for $t>t_{\max }$ is similar that found in $\S 3$ (see figure $1 a$ ), it is more surprising here since under the strain for $t<t_{\max }$ more enstrophy than scalar variance was transferred into azimuthal components. In short, the enstrophy overshoots the scalar when the strain is switched on, but then rebounds to lower values when it is switched off. For comparison, the Kida (1981) vortex patch oscillates between circular and elliptical states for $0<t<t_{\max }$; when the strain is turned off the vortex will be left in whatever state it happens to be at $t_{\max }$ and will rotate steadily thereafter. No enstrophy can be lost to fine scales in this case, and the vortex cannot relax to axisymmetry.

Analytically only the passive scalar may be handled; for $t>t_{\max }$ the solution for $\sigma_{1}$ is

$$
\sigma_{1}=\left(\mathrm{e}^{-\mathrm{i} n \alpha\left(t-t_{\max }\right)}-\mathrm{e}^{-\mathrm{i} n \alpha t}\right) \Sigma_{1}(r) .
$$

This relaxes weakly back to zero as $t \rightarrow \infty$, as does the vorticity field $\omega_{1}$. For $\sigma_{2}$ we have

$$
\sigma_{2}=r^{-1} \partial_{r}\left[2 \phi^{2} \beta \alpha^{-2}\left(1-\cos n \alpha t_{\max }\right)\right],
$$

and the weak convergence of $\sigma_{2}$ to $\Sigma_{2}$ is arrested.

\section{Behaviour under a random forcing}

We have seen that the vorticity distribution is more robust than the passive scalar, with relatively little enstrophy lost to small scales and dissipation. We have also noted that the effect of an external step input on the mean scalar field may be written as a diffusion operator (plus fluctuations). In this section we present preliminary investigations of the diffusive spreading of vorticity or a passive scalar in the presence of a continuous random external input. We take $q(t)$ to be a random stationary Gaussian function with the correlation function

$$
\left\langle q(t) q^{*}\left(t^{\prime}\right)\right\rangle=c\left(t-t^{\prime}\right), \quad c(t)=\exp \left(-t^{2} / 2 t_{\text {corr }}^{2}\right),
$$

where $\langle\cdot\rangle$ is an ensemble average and $q(t)$ varies on a correlation time scale given by $t_{\text {corr }}$.

For our original example of a vortex moving in two-dimensional turbulence in $\S 1$, the straining $q(t)$ experienced by a given vortex has a magnitude $l^{2} / L^{2}=\varepsilon$ and time scale $t_{\text {corr }}=1 / \varepsilon$, and will effectively be random if the configuration contains many vortices moving in the plane. If instead there are only a few vortices in a periodic or quasi-periodic motion, $q(t)$ will contain only a limited number of frequencies and not be random. For the passive scalar problem such a deterministic perturbation leads to the creation of narrow islands at resonant surfaces of the basic flow given by $\psi_{0}$, and no systematic diffusion for small $\varepsilon$ (see, for example, papers in the collection Mackay $\&$ Meiss 1987). To obtain systematic diffusion within our perturbative framework, we take $q(t)$ to be a random function, modelling the motion of many external vortices.

\subsection{Governing system and invariants}

We now allow the $O\left(\varepsilon^{2}\right)$ changes to the mean profile in $\omega_{2}$ to affect the leading-order mean profile $\omega_{0}$, in order to follow the diffusion of the vorticity field. In the expansion 
(2.5) we subsume $\varepsilon^{2} \omega_{2}$ into $\omega_{0}$ and absorb the source terms in the equation (2.6e) for $\omega_{2}$ into the equation $(2.6 a)$ for $\omega_{0}$ to give the system

$$
\begin{aligned}
\partial_{t} \omega_{0}+\mathrm{i} n \varepsilon^{2} r^{-1} \partial_{r}\left[\left(\psi_{1}+q \phi\right) \omega_{1}^{*}\right]+\text { c.c. } & =R e^{-1} \Delta_{0} \omega_{0}, \\
\partial_{t} \omega_{1}+\mathrm{i} n \alpha \omega_{1}+\mathrm{i} n \beta\left(\psi_{1}+q \phi\right) & =\operatorname{Re}^{-1} \Delta_{1} \omega_{1},
\end{aligned}
$$

with $(2.6 b, d)$ and $(2.7)$ holding as before. This is essentially a multiple-scale expansion; the source terms now appearing in the $\omega_{0}$ equation lead to evolution of the mean profile over a long $O\left(\varepsilon^{-2}\right)$ time scale. However as we cannot deal with this system analytically, the small parameter $\varepsilon$ remains explicitly. Note that this system, consisting of the $n=2$ mode and mean field, is now more in the nature of a truncation of the full dynamics than the systems studied in $\S \S 3,4$. For example, it is possible that the mean vorticity field could evolve over $O\left(\varepsilon^{-2}\right)$ time scales so as to become unstable to an $n=3$ mode, but this mode is not present.

We will focus on the enstrophy in the system, which may now be written

$$
\begin{aligned}
E_{\omega} & =E_{\omega}^{\text {mean }}+\varepsilon^{2} e_{\omega}^{\text {azi }}+O\left(\varepsilon^{4}\right), \\
E_{\omega}^{\text {mean }} & =\frac{1}{2} \int_{0}^{\infty} \omega_{0}^{2} 2 \pi r \mathrm{~d} r, \quad e_{\omega}^{\text {azi }}=\int_{0}^{\infty}\left|\omega_{1}\right|^{2} 2 \pi r \mathrm{~d} r .
\end{aligned}
$$

For the analogous scalar problem, the system is

$$
\begin{aligned}
\partial_{t} \sigma_{0}+\mathrm{i} n \varepsilon^{2} r^{-1} \partial_{r}\left(q \phi \sigma_{1}^{*}\right)+\text { c.c. } & =P e^{-1} \Delta_{0} \sigma_{0}, \\
\partial_{t} \sigma_{1}+\mathrm{i} n \alpha \sigma_{1}+\mathrm{i} n \beta q \phi & =P e^{-1} \Delta_{1} \sigma_{1},
\end{aligned}
$$

with (2.18) holding as before. The mean and azimuthal scalar variances $E_{\sigma}^{\text {mean }}$ and $e_{\sigma}^{\text {azi }}$ are defined analogously to $(5.3 b, c)$.

For the vorticity problem we specify the initial condition (2.8); for the analogous scalar problem we take $\psi_{0}$ given by $(2.8 b)$ and $\sigma_{0}$ by (2.19). Now that the basic vorticity profile $\omega_{0}$ and scalar profile $\sigma_{0}$ are allowed to evolve through the new source terms in (5.2a) and (5.4a) it is important to note that the flow field $\psi_{0}$ acquires a different status in the two problems. In the passive scalar problem it is fixed for all time, while for vorticity it is coupled to $\omega_{0}$ and so evolves (and generally weakens) as the vorticity spreads out. Only for moderate times will the underlying axisymmetric flows $\psi_{0}$ be similar in the two problems.

\subsection{Quasi-linear theory for a passive scalar}

We begin by deriving a quasi-linear approximation for the diffusion of the passive scalar (Bazzani, Siboni \& Turchetti 1994) to obtain an eddy diffusivity $\kappa(r)$. This calculation quantifies the interaction between the basic flow specified by $\psi_{0}$ and the external straining given by the statistics of $q(t)$. Our derivation is not rigorous, but is corroborated numerically below. We solve $(5.4 b)$ (with $P e=\infty$ ) for any $q(t)$ as

$$
\sigma_{1}=-\mathrm{i} n \beta \phi \int_{0}^{t} \mathrm{e}^{-\mathrm{in} \alpha(t-\tau)} q(\tau) \mathrm{d} \tau .
$$

Substituting this into $(5.4 a)$ yields

$$
\partial_{t} \sigma_{0}=r^{-1} \partial_{r}\left(\varepsilon^{2} n^{2} \phi^{2} \beta \int_{0}^{t} q(t) q^{*}(\tau) \mathrm{e}^{\mathrm{i} n \alpha(t-\tau)} \mathrm{d} \tau+\text { c.c. }\right)+P e^{-1} \Delta_{0} \sigma_{0} .
$$




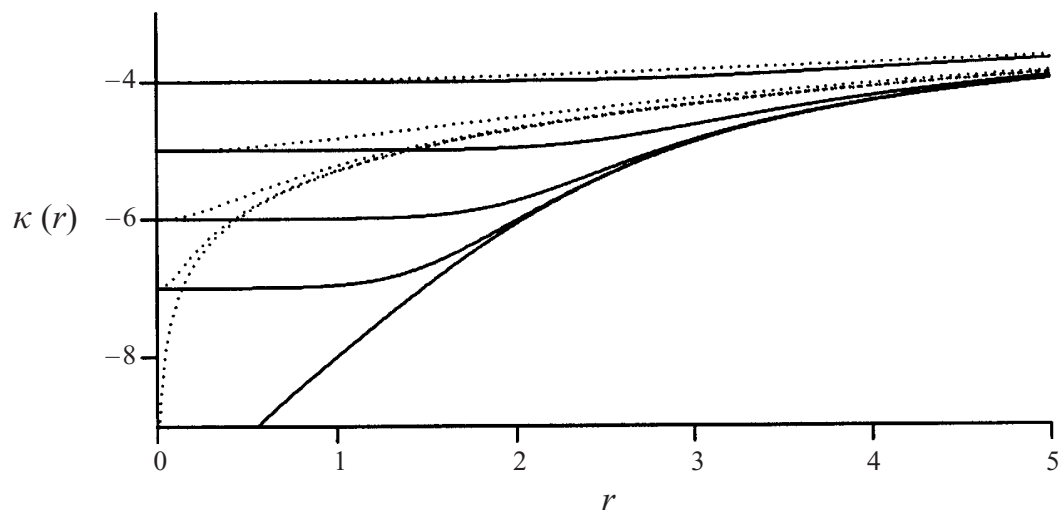

FIGURE 8. Effective scalar diffusivity $\kappa(r)$ plotted against $r$ for $P e=10^{4}, 10^{5}, 10^{6}, 10^{7}$ and $\infty$ (solid curves, reading downward). Parameter values are $t_{\text {corr }}=50$ and $\varepsilon=10^{-4}$. The dotted curves show $\kappa(r)$ in the absence of vortex motion, $\alpha(r) \equiv 0$, for the same values of $P e$.

If $q(t) q^{*}(\tau)$ is averaged over realizations (using (5.1)) and $\beta=r^{-1} \partial_{r} \sigma_{0}$ substituted, we obtain the diffusion equation

$$
\partial_{t} \sigma_{0}=r^{-1} \partial_{r}\left(r \kappa(r) \partial_{r} \sigma_{0}\right)
$$

with the space-dependent diffusivity given by

$$
\kappa(r)=\varepsilon^{2} n^{2} \phi^{2} r^{-2} \int_{0}^{\infty} c(s) \mathrm{e}^{\mathrm{i} n \alpha(r) s} \mathrm{~d} s+\text { c.c. }+P e^{-1},
$$

for large $t$, so that the lower limit in (5.6) is irrelevant. Note that in ensembleaveraging (5.6) we identified $\sigma_{0}$ with the ensemble-averaged mean scalar field: while this assumption is reasonable, a rigorous justification is delicate (see discussion in Bazzani et al. 1994) and we do not take up this issue here. Note also that it is legitimate to neglect $P e$ in obtaining (5.5) (while keeping it in (5.6)) provided the shear-diffuse time scale (2.22) is longer than the correlation time.

For the Gaussian correlation function (5.1),

$$
\kappa(r)=\sqrt{2 \pi} \frac{\varepsilon^{2} n^{2} \phi^{2} t_{\text {corr }}}{r^{2}} \mathrm{e}^{-n^{2} \alpha^{2} t_{\text {corr }}^{2} / 2}+P e^{-1} .
$$

The effective diffusivity $\kappa(r)$ depends on the correlation time $t_{\text {corr }}$ and the flow, through the angular velocity $\alpha(r)$. For small correlation times with $\alpha t_{\text {corr }} \ll 1, \kappa$ has no dependence on the flow field at all. For large $t_{\text {corr }}$ and $P e$ the influence of the flow is very important, and in regions where $\alpha t_{\text {corr }} \gg 1, \kappa$ is vanishingly small: here the fast angular motion of particles compared with the time scale $t_{\text {corr }}$ leads to an averaging of the external strain, and hence a reduction in its effect.

To illustrate this, we take $t_{\text {corr }}=50, \varepsilon=10^{-4}$ and in figure 8 plot $\log _{10} \kappa$ against $r$ for $P e=10^{4}, 10^{5}, 10^{6}, 10^{7}$ and $\infty$, reading downwards (solid curves). As $P e$ is increased, the diffusion near the centre of the vortex is suppressed. To illustrate the role of the underlying vortex, the dotted lines show the effective diffusivity $\kappa$ for the same values of $P e$ in the case of no vortex motion, i.e. only the random strain field acting and $\alpha(r)=0$ in (5.9). Note that for an exponential correlation function $c(t)=\mathrm{e}^{-|t| / t_{\text {corr }}}$ the effective diffusivity is

$$
\kappa=\frac{2 \varepsilon^{2} n^{2} \phi^{2} t_{\text {corr }}}{r^{2}\left(1+n^{2} \alpha^{2} t_{\text {corr }}^{2}\right)}+P e^{-1}
$$

and is similar to that found in (4.9) for the step-function input. 


\subsection{Comparison of vorticity and passive scalar}

For vorticity it is not possible to solve $(5.2 b)$ and so we cannot compute explicitly the effect of eddy diffusion. To see how vorticity behaves, the governing systems (5.2) and (5.4) were solved numerically using boundary conditions and methods similar to those described above in $\S 2.3$. To realize the random input $q(t)$ numerically we follow a much simplified version of the procedure of Kraichnan (1970) and Drummond, Duane \& Horgan (1984), and set

$$
q(t)=M^{-1 / 2} \sum_{m=1}^{M} \exp \left(\mathrm{i} \zeta_{m} t+\mathrm{i} \phi_{m}\right),
$$

where the coefficients $\zeta_{m}$ are chosen randomly from a Gaussian distribution with variance $1 / t_{\text {corr }}^{2}$ and mean zero, and $\phi_{m}$ are uniformly distributed in the interval $(0,2 \pi)$. Such a $q(t)$ is a smooth function which varies on the time scale $t_{\text {corr }}$; in fact one can check that $\left\langle\left|q^{\prime}(t)\right|^{2}\right\rangle=t_{\text {corr }}^{-2}$. We take $M=1000$ and in order to test and to compare results, we use the same realization of the random function $q(t)$ for all the runs below.

In the absence of viscosity and scalar diffusion, once the statistics of $q(t)$ and the structure of the initial vortex are specified by (5.1) and (2.8), the only parameters remaining are the strength $\varepsilon$ and correlation time $t_{\text {corr }}$ of the external strain. We require $\varepsilon$ to be small for our perturbative approach to be valid, and also to avoid numerical instability; we fix $\varepsilon=10^{-4}$ in the runs below. In terms of our original discussion of the random straining arising from many other vortices, the appropriate correlation time is $t_{\text {corr }}=O(1 / \varepsilon)=O\left(10^{4}\right)$; however then it would be impossible to follow the fields numerically for more than a few correlation times. We are therefore constrained to take a rather smaller value for $t_{\text {corr }}$, and fix $t_{\text {corr }}=50$ below, which is of similar magnitude to the turnover time scale of the vortex. For numerical reasons it is also necessary to introduce molecular diffusion of scalar or vorticity, in order to damp down the small scales that are generated over the long runs below (unlike in the relatively short runs of $\S \S 3,4)$. We take $R e$ and $P e$ in the range $10^{4}-10^{7}$ in the simulations, which last between $10^{4}$ and $10^{5}$ time units.

In figure $9(a), R e=P e=10^{4}$ : the upper solid curve shows the mean enstrophy $E_{\omega}^{\text {mean }}$, and the lower solid curve the scalar variance $E_{\sigma}^{\text {mean }}$, as functions of time. The dashed curve shows the quasi-linear result for the scalar variance, obtained by integrating equation (5.7) numerically. These curves have been separated by subtracting constants from the data, otherwise they would intersect the vertical axis at the same point, and overlap. This figure shows that the quasi-linear theory (dashed) gives good agreement with the scalar variance (lower solid); the behaviour of the vorticity (upper solid) is very similar.

This run is relatively diffusive, and a major fraction of the enstrophy and scalar variance is destroyed. Also, purely diffusive spreading of the Gaussian vortex profile is a significant effect as the run goes up to $t=2 \times 10^{4}=2 T_{\text {mean }}$ (recall equation (2.21)). The shear-diffuse time scale (2.22) here is only moderate at $T_{\text {azi }} \simeq 430$ and this appears to be the reason why vorticity and scalar behave so similarly: the rebound of enstrophy from azimuthal to mean part is arrested because of the diffusive decay (and rearrangement) of the azimuthal field. Note also from figure 8 that at this low value of $P e$ the effective scalar diffusivity (upper solid curve) differs little from the effective diffusivity in the absence of any vortex motion (upper dotted curve), which is another reason why one might not expect scalar and vorticity to behave differently at such low $R e$ and $P e$. 

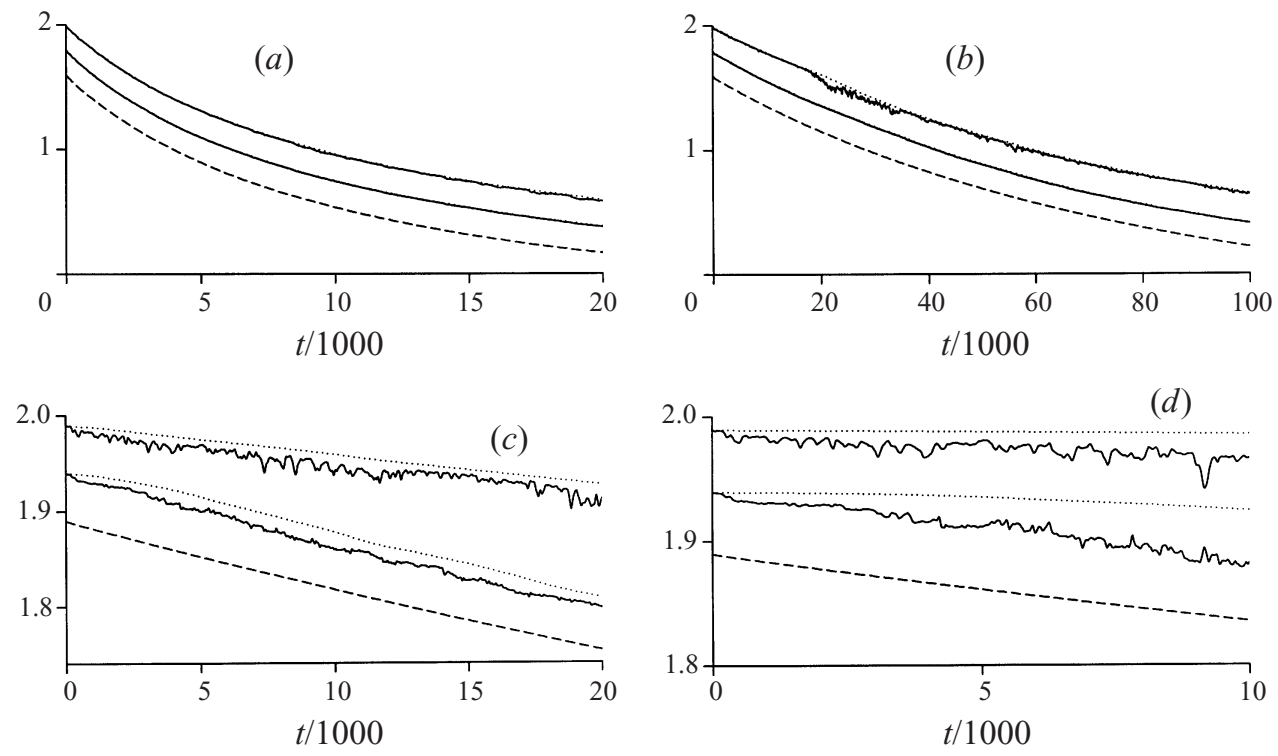

FiguRE 9. Mean enstrophy $E_{\omega}^{\text {mean }}$ (upper solid), total enstrophy $E_{\omega}^{\text {mean }}+\varepsilon^{2} e_{\omega}^{\text {azi }}$ (upper dotted), mean scalar variance $E_{\sigma}^{\text {mean }}$ (lower solid), total scalar variance $E_{\sigma}^{\text {mean }}+\varepsilon^{2} e_{\sigma}^{\text {azi }}$ (lower dotted), and quasi-linear results for $E_{\sigma}^{\text {mean }}$ (dashed), multiplied by 100 , are plotted against $t / 1000$ for $(a) R e=P e=10^{4}$, (b) $R e=P e=10^{5}$, (c) $R e=P e=10^{6}$ and (d) $R e=P e=10^{7}$. Constants are subtracted to separate the curves in each figure.

Differences between vorticity and scalar only begin to emerge as molecular diffusion is reduced further. This may be seen in figure $9(b)$ which shows a run with $R e=$ $P e=10^{5}$ for $0 \leqslant t \leqslant 10^{5}$. Up to time $t=2 \times 10^{4}$ the level of mean enstrophy (upper solid) decays at a slower rate than the mean scalar variance (lower solid). At later times the enstrophy begins to dissipate more rapidly: this is to be expected since we recall that the stream function $\psi_{0}$ for the vorticity problem is coupled to the vorticity distribution, and as it spreads out, the flow will become weaker and, based on the behaviour of the scalar, we may expect the effective diffusivity to become stronger (see (5.9)). Even with this effect, though, the net loss of enstrophy by the end of the run is marginally less than that of the scalar variance.

The difference between scalar and vorticity is most marked in the shorter runs shown in figure $9(c)$ with $R e=P e=10^{6}$ and figure $9(d)$ with $R e=P e=10^{7}$. Again the upper solid curve shows $E_{\omega}^{\text {mean }}$, the lower solid curve $E_{\sigma}^{\text {mean }}$, and the dashed curve the quasi-linear result. Also plotted are the total enstrophy $E_{\omega}^{\text {mean }}+\varepsilon^{2} e_{\omega}^{\text {azi }}$ (upper dotted curve) and total scalar variance $E_{\sigma}^{\text {mean }}+\varepsilon^{2} e_{\sigma}^{\text {azi }}$ (lower dotted curve); these decay smoothly, confirming that the fluctuations in the mean (solid curves) arise from transfers between mean and azimuthal components.

As $R e=P e$ is increased, the decay of both enstrophy and scalar variance slows down markedly: note the change in vertical scales between figure $9(a-d)$. While the scalar and quasi-linear results remain in good agreement, the decay of enstrophy becomes significantly slower. Figure $9(d)$ represents our closest approach to truly inviscid or non-diffusive behaviour. In this figure the quasi-linear results (dashed) with $P e=10^{7}$ are indistinguishable from those for $P e=\infty$ (not shown) and are in good agreement with the scalar results (lower solid curve). Thus the behaviour of the mean scalar variance $E_{\sigma}^{\text {mean }}$ (lower solid curve) appears to have achieved the 

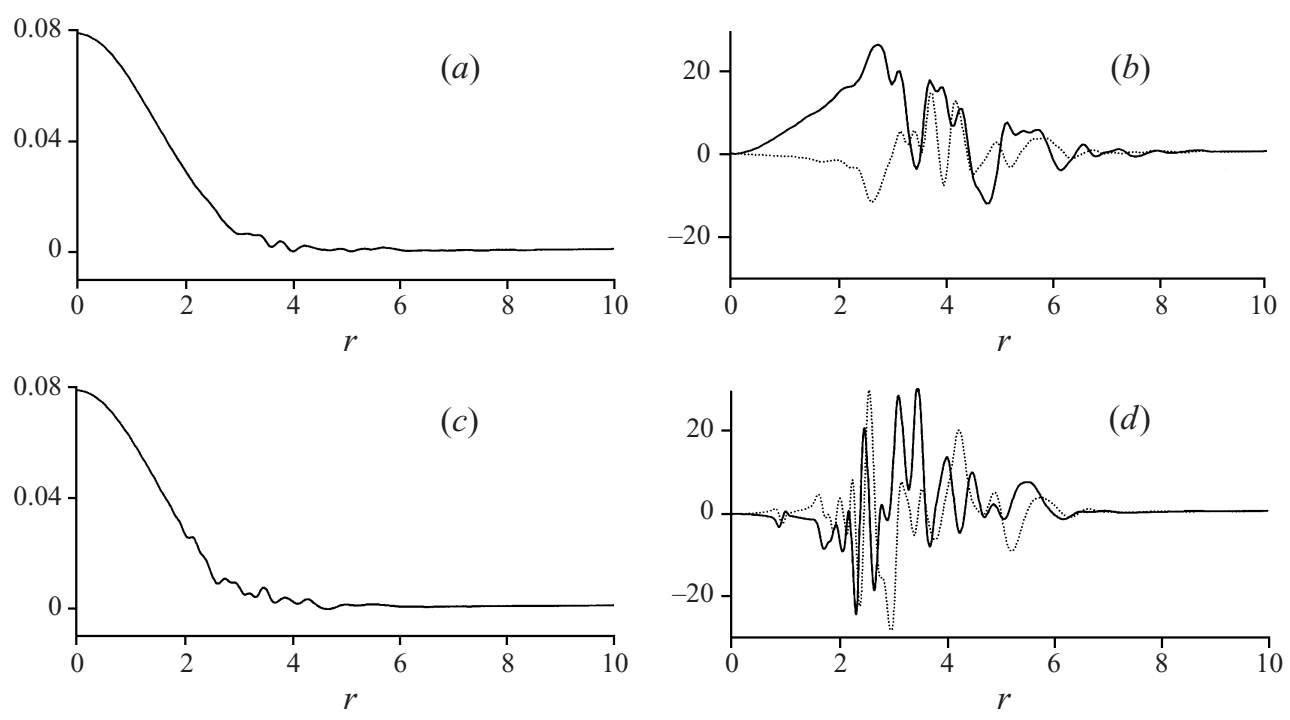

Figure 10. (a) $\omega_{0}$, (b) $\omega_{1},(c) \sigma_{0}$ and $(d) \sigma_{1}$ are plotted against $r$ for $R e=P e=10^{6}$ and $t=10^{4}$. Real parts are shown solid, imaginary parts dotted.

non-diffusive limit. While we have no inviscid theory against which to check the behaviour of enstrophy at $R e=10^{7}$, by comparison with the scalar (and figure $9 \mathrm{c}$ ), we are confident that relatively slow decay of enstrophy shown in figure $9(d)$ is a feature of the inviscid dynamics. As well as slower decay, the enstrophy shows more variation on short time scales than the scalar variance, a behaviour in agreement with the discussion in $\S 4.1$, the mechanism depicted in figure 5, and the results of Ohkitani (1991).

Note that were there truly zero diffusion in figure $9(d)$ (and we were not using a perturbation expansion) the dotted lines would be flat, representing constant total enstrophy; the decay in the mean would then simply represent a transfer from the mean to the azimuthal component and subsequent, indefinite, fine-scaling by differential rotation.

At the largest values of $R e$ and $P e$ our runs are far too short to show the long-time behaviour, and in fact the spreading of the vorticity or scalar field is really occurring only at the edge of the vortex. This is in agreement with figure 8 , which shows that at this value of $P e$ the effective diffusivity (fourth solid curve, reading downwards) is extremely small except near the vortex edge at $r \gtrsim 2$. Figures $10(a)$ and $10(b)$ show the mean and azimuthal vorticity respectively for $R e=10^{6}$ and $t=10^{4}$; figures $10(c)$ and $10(d)$ show the same for the scalar. The generation of fine structure in both fields is more marked for the scalar than for the vorticity.

\section{Discussion}

We have studied the response of a Gaussian vortex to weak, externally imposed strain flows of various forms. Our main result is the presence of a 'rebound' phenomenon, whereby vorticity is transferred from the azimuthal component back to the mean, axisymmetric component, during the process of spiral wind-up. The vortex is much more robust than an analogous passive scalar distribution: because of the rebound mechanism, lower levels of enstrophy than scalar variance are transferred 
into azimuthal components and so then lost to fine scales, and dissipation by the shear-diffuse mechanism for finite $R e$ or $P e$. This was seen for all the strain flows studied, at sufficiently large Reynolds number, and some theoretical support was obtained using Lundgren's long-time asymptotic solution for spiral wind-up.

It appears that not only are there wide classes of vortices that are linearly and nonlinearly stable (e.g. Gent \& McWilliams 1986; Dritschel 1988), but that the internal dynamics of a vortex can to some extent resist the effect of strain in entraining irrotational fluid within the vortex, and so resist the spreading and weakening of the vortex. This behaviour is reminiscent of Kida's (1981) vortex patch solution: in this exact solution, the vortex remains elliptical, behaving reversibly and showing perfectly elastic behaviour. There is no mixing of the vortex with the surrounding fluid. Although the smooth Gaussian vortex shows damped, rather than elastic, behaviour and tends to relax to a steady state through the generation of fine structure in the vortex, nevertheless the core of the vortex shows striking integrity. Disturbances tend to be suppressed, especially near the centre, and also to propagate outwards towards the periphery of the vortex (Montgomery \& Kallenbach 1997).

The main restriction in the present paper is that we consider only weak external strain, using small values of the parameter $\varepsilon$. This perturbative approach allows us to separate the transfers of enstrophy in a clean way, and in $\S \S 3,4$ to run simulations at sufficiently high Reynolds number that they are essentially inviscid, despite the generation of fine structure. Nevertheless the small values of $\varepsilon$ are rather restrictive when a continuous random strain field is applied, and do not allow us to follow the decay of a vortex at high $R e$ for long times. We are now studying the evolution of vortices in stronger external strain fields, using a fully two-dimensional code: preliminary results confirm the rebound phenomenon discussed in this paper.

One process that can become important for larger values of $\varepsilon$ is 'vortex stripping' (see Legras \& Dritschel 1993), in which a vortex loses vorticity from its periphery and is left with a sharp edge. This process can readily occur during close vortex encounters in two-dimensional turbulence, and such sharp-edged vortices appear then to be able to resist the relaxation to axisymmetry that appears to occur for the Gaussian vortex (Dritschel 1998). It would be of interest to extend the present study (and BG98) to vortices with sharp edges. The question then is whether the rebound phenomenon occurs in this case, and whether the persistent non-axisymmetrization observed is present in linear theory, or is an intrinsically nonlinear effect.

We should also note that the spreading of the passive scalar under random strain computed in (5.9) is very small for large $t_{\text {corr }}$, and so too presumably is the spreading of a Gaussian vortex. In particular for a very dilute two-dimensional turbulence of similar-sized vortices, with $\varepsilon \ll 1$ and $t_{\text {corr }}=O\left(\varepsilon^{-1}\right)$, the corresponding scalar diffusivity decreases with $\varepsilon$ faster than any power law. This suggests that the diffusive spreading of vortices is a slow effect compared with vortex collisions, which presumably scale as some power law in $\varepsilon$, even if three rather than two vortices are involved in typical collisions (e.g. Dritschel 1995). This conclusion is however again based on a Gaussian vortex, and should be re-examined for vortices with sharper edges, more characteristic of two-dimensional turbulence.

We are grateful to Professor D. G. Dritschel and Professor C. A. Jones for useful discussions. We should like to thank Professor M. T. Montgomery for bringing several important references to our attention, and the referees for a number of useful points and references.

This work was revised while A.B. was at the School of Mathematics, University of 
New South Wales. Thanks are due to the Australian Research Council and the Royal Society for grants which made this extended visit possible. Gratitude is also expressed to both the staff of the School (especially Peter Blennerhassett) and to the staff and students of New College, UNSW for their hospitality.

\section{REFERENCES}

Andreotti, B., Doundy, S. \& Couder, Y. 1997 About the interaction between vorticity and stretching in coherent structures. In Turbulence Modelling and Vortex Dynamics, vol. 491 (ed. O. Boratav, A. Eden \& A. Erzan), pp. 92-108. Springer.

AreF, H. 1984 Stirring by chaotic advection. J. Fluid Mech. 143, 1-21.

Babiano, A., Basdevant, C., Legras, B. \& Sadourny, R. 1987 Vorticity and passive scalar dynamics in two-dimensional turbulence. J. Fluid Mech. 183, 379-397.

Bassom, A. P. \& GilberT, A. D. 1998 The spiral wind-up of vorticity in an inviscid planar vortex. J. Fluid Mech. 371, 109-140 (referred herein to as BG98).

Bazzani, A., Siboni, S. \& Turchetti, G. 1994 Diffusion in Hamiltonian systems with a small stochastic perturbation. Physica D 76, 8-21.

Bernoff, A. J. \& Lingevitch, J. F. 1994 Rapid relaxation of an axisymmetric vortex. Phys. Fluids 6, 3717-3723 (referred to herein as BL94).

Brachet, M. E., Meneguzzi, M., Politano, H. \& Sulem, P.-L. 1988 The dynamics of freely decaying two-dimensional turbulence. J. Fluid Mech. 194, 333-349.

Briggs, R. J., Daugherty, J. D. \& Levy, R. H. 1970 Role of Landau damping in crossed-field electron beams and inviscid shear flow. Phys. Fluids 13, 421-432.

DritsChel, D. G. 1988 Nonlinear stability bounds for inviscid, two-dimensional, parallel or circular flows with monotonic vorticity, and the analogous three-dimensional quasi-geostrophic flows. J. Fluid Mech. 191, 575-581.

Dritschel, D. G. 1989a Contour dynamics and contour surgery: numerical algorithms for extended high-resolution modelling of vortex dynamics in two-dimensional, inviscid, incompressible flows. Comput. Phys. Rep. 10, 77-146.

Dritschel, D. G. $1989 \mathrm{~b}$ On the stabilization of a two-dimensional vortex strip by adverse shear. J. Fluid Mech. 206, 193-221.

DritsChel, D. G. 1990 The stability of elliptical vortices in an external straining flow. J. Fluid Mech. 210, 223-261.

Dritschel, D. G. 1995 A general theory for two-dimensional vortex interactions. J. Fluid Mech. 293, 269-303.

DrITSCHEL, D. G. 1998 On the persistence of non-axisymmetric vortices in inviscid two-dimensional flows. J. Fluid Mech. 371, 141-155.

Dritschel, D. G., Haynes, P. H., Juckes, M. N. \& Shepherd, T. G. 1991 The stability of a two-dimensional vorticity filament under uniform strain. J. Fluid Mech. 230, 647-665.

Drummond, I. T., Duane, S. \& Horgan, R. R. 1984 Scalar diffusion in simulated helical turbulence with molecular diffusivity. J. Fluid Mech. 138, 75-91.

Fornberg, B. 1977 A numerical study of 2-d turbulence. J. Comput. Phys. 25, 1-31.

Gent, P. R. \& McWilliams, J. C. 1986 The instability of barotropic circular vortices. Geophys. Astrophys. Fluid Dyn. 35, 209-233.

JimÉnez, J., MofFATt, H. K. \& VASCO, C. 1996 The structure of vortices in freely decaying twodimensional turbulence. J. Fluid Mech. 313, 209-222.

Keller, H. B. 1971 A new difference scheme for parabolic problems. In Numerical Solutions of Partial Differential Equations (ed. B. Hubbard), vol. 2, pp. 327-350. Academic.

Kevlahan, N. K.-R. \& Farge, M. 1997 Vorticity filaments in two-dimensional turbulence: creation, stability and effect. J. Fluid Mech. 346, 49-76.

KIDA, S. 1981 Motion of an elliptic vortex in a uniform shear flow. J. Phys. Soc. Japan 50, 3517-3520.

Koumoutsakos, P. 1997 Inviscid axisymmetrization of an elliptical vortex. J. Comput. Phys. 138, 821-857.

Kraichnan, R. H. 1970 Diffusion by a random velocity field. Phys. Fluids 13, 22-31.

LEgRAS, B. \& DRITSChEL, D. G. 1993 Vortex stripping and the generation of high vorticity gradients in two-dimensional flows. Appl. Sci. Res. 51, 445-455. 
Lingevitch, J. F. \& Bernoff, A. J. 1995 Distortion and evolution of a localized vortex in an irrotational flow. Phys. Fluids 7, 1015-1026.

Llewellyn Smith, S. G. 1995 The influence of circulation on the stability of vortices to mode-one disturbances. Proc. R. Soc. Lond. A 451, 747-755.

Lundgren, T. S. 1982 Strained spiral vortex model for turbulent fine structure. Phys. Fluids 25, 2193-2203.

Mackay, R. S. \& Meiss, J. D. 1987 Hamiltonian Dynamical Systems. Adam Hilger.

MCCALPIn, J. D. 1987 On the adjustment of azimuthally perturbed vortices. J. Geophys. Res. C 92, 8213-8225.

McWilliams, J. C. 1984 The emergence of isolated coherent vortices in turbulent flow. J. Fluid Mech. 146, 21-43.

Melander, M. V., McWilliams, J. C. \& Zabusky, N. J. 1987 Axisymmetrization and vorticitygradient intensification of an isolated two-dimensional vortex through filamentation. J. Fluid Mech. 178, 137-159.

Moffatt, H. K. \& Kamkar, H. 1983 On the time-scale associated with flux expulsion. In: Stellar and Planetary Magnetism (ed. A. M. Soward), pp. 91-97. Gordon \& Breach.

Moffatt, H. K., Kida, S. \& OHKITANi, K. 1994 Stretched vortices - the sinews of turbulence: high Reynolds number asymptotics. J. Fluid Mech. 259, 241-264.

Montgomery, M. T. \& Kallenbach, R. J. 1997 A theory for vortex Rossby-waves and its application to spiral bands and intensity changes in hurricanes. Q. J. R. Met. Soc. 123, 435-465.

Moore, D. W. \& Saffman, P. G. 1971 Structure of a line vortex in an imposed strain. In Aircraft Wake Turbulence and its Detection (ed. J. H. Olson, A. Goldburg \& M. Rogers), pp. 339-354. Plenum.

Oetzel, K. G. \& Vallis, G. K. 1997 Strain, vortices, and the enstrophy inertial range in twodimensional turbulence. Phys. Fluids 9, 2991-3004.

OHKITANi, K. 1991 Wave number space dynamics of enstrophy cascade in a forced two-dimensional turbulence. Phys. Fluids A 3, 1598-1611.

Ottino, J. M. 1989 The Kinematics of Mixing: Stretching, Chaos and Transport. Cambridge University Press.

Rhines, P. B. \& Young, W. R. 1982 Homogenization of potential vorticity in planetary gyres. J. Fluid Mech. 122, 347-367.

Rhines, P. B. \& Young, W. R. 1983 How rapidly is a passive scalar mixed within closed streamlines? J. Fluid Mech. 133, 133-145.

Robert, R. \& Sommeria, J. 1991 Statistical equilibrium states for two-dimensional flows. J. Fluid Mech. 229, 291-310.

Robinson, A. C. \& Saffman, P. G. 1984 Stability and structure of stretched vortices. Stud. Appl. Maths 70, 163-181.

Rossi, L. F., Lingevitch, J. F. \& Bernoff, A. J. 1997 Quasi-steady monopole and tripole attractors for relaxing vortices. Phys. Fluids 9, 2329-2338.

Smith, G. B. \& Montgomery, M. T. 1995 Vortex axisymmetrization: dependence on azimuthal wave-number or asymmetric radial structure changes. Q. J. R. Met. Soc. 121, 1615-1650.

Smith, R. A. \& Rosenbluth, M. N. 1990 Algebraic instability of hollow electron columns and cylindrical vortices. Phys. Rev. Lett. 64, 649-652.

Ting, L. \& Klein, R. 1991 Viscous Vortical Flows. Lecture Notes in Physics, vol. 374. Springer.

VAssilicos, J. C. 1995 Anomalous diffusion of isolated flow singularities and of fractal or spiral structures. Phys. Rev. E 52, R5753-R5756.

Weiss, N. O. 1966 The expulsion of magnetic flux by eddies. Proc. R. Soc. Lond. A 293, 310-328.

YAO, H. B. \& ZABUSKY, N. J. 1996 Axisymmetrization of an isolated vortex region by splitting and partial merging of satellite depletion perturbations. Phys. Fluids 8, 1842-1847. 